\title{
Shearer's measure and stochastic domination of product measures
}

\author{
Christoph Temmel *†
}

\begin{abstract}
Let $G:=(V, E)$ be a locally finite graph. Let $\vec{p} \in[0,1]^{V}$. We show that Shearer's measure, introduced in the context of the Lovász Local Lemma, with marginal distribution determined by $\vec{p}$, exists on $G$ iff every Bernoulli random field with the same marginals and dependency graph $G$ dominates stochastically a non-trivial Bernoulli product field. Additionally we derive a non-trivial uniform lower bound for the parameter vector of the dominated Bernoulli product field. This generalises previous results by Liggett, Schonmann \& Stacey in the homogeneous case, in particular on the $k$-fuzz of $\mathbb{Z}$. Using the connection between Shearer's measure and a hardcore lattice gas established by Scott \& Sokal, we transfer bounds derived from cluster expansions of lattice gas partition functions to the stochastic domination problem.
\end{abstract}

Keywords: stochastic domination, Lovász Local Lemma, product measure, Bernoulli random field, stochastic order, hardcore lattice gas.

MSC 2010: 60E15 (primary), 60G60, 82B20, 05D40.

This is an extended version of [21].

\section{Contents}

2 Setup and problem statement 3

3 A primer on Shearer's measure 5

4 Main results and discussion $\ldots \ldots \ldots$

4.1 Reinterpretation of bounds . . . . . . . . . . . . 8

*5030 Institut für Mathematische Strukturtheorie, Technische Universität Graz, Steyrergasse 30/III, 8010 Graz, Austria

${ }^{\dagger}$ Email: temmel@math.tugraz.at 
5 Proofs 9

5.1 Tools for stochastic domination . . . . . . . . . . . . . . . . 10

5.2 Nondomination . . . . . . . . . . . . . . . . . 11

5.3 One vertex open extension probabilities . . . . . . . . . . 11

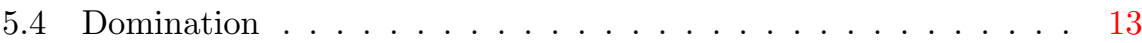

6 The weak invariant case 16

7 The asymptotic size of the jump on $\mathbb{Z}_{(k)} \quad 17$

8 Additional Material 20

8.1 Intrinsic coupling and domination of Shearer's measure . . . . . . 20

8.2 Tools for stochastic domination II . . . . . . . . . . . . . . . . . . 23

8.3 A summary of the homogeneous case . . . . . . . . . . . . 26

8.4 Proofs of classical results . . . . . . . . . . . . . . . . . 28

\section{List of Figures}

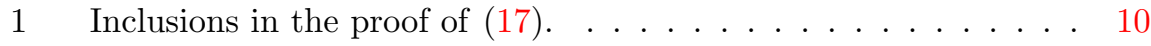

2 Inequalities in the proof of $(55) \ldots \ldots . \ldots 28$

\section{Introduction}

The question under which conditions a Bernoulli random field (short BRF) stochastically dominates a Bernoulli product field (short BPF) is of interest in probability and percolation theory. Knowledge of this kind allows the transfer of results from the independent case to more general settings. Of particular interest are BRFs with a dependency structure described by a graph $G$ and prescribed common marginal parameter $p$, as they often arise from rescaling arguments [11], dependent models [6] or particle systems [14]. In this setting an interesting question is to find lower bounds on $p$ which guarantee stochastic domination for every such BRF.

This question has been investigated in the setting of boot-strap percolation [1, section 2] and super-critical Bernoulli percolation [2, section 2]. Finally Liggett, Schonmann \& Stacey [15] derived a generic lower bound for dependency graphs with uniformly bounded degree. Of particular interest is the $k$-fuzz of $\mathbb{Z}$ ( short $\mathbb{Z}_{(k)}$, that is the graph with vertex set $\mathbb{Z}$ and edges between all integers at distance less than or equal to $k$ ), which is the dependency graph of $k$-dependent BRFs on $\mathbb{Z}$. In this case they determined the minimal $p$, for which stochastic domination of a non-trivial BPF holds for each such BRF on $\mathbb{Z}_{(k)}$. Even more, they showed, that in this case the parameter of the dominated BPF is uniformly bounded from below and nonzero for this minimal $p$ and made a conjecture about the size of the jump of the value of the parameter of the dominated $\mathrm{BPF}$ at this minimal $p$.

Their main tools have been a sufficient condition highly reminiscent of the Lovász Local Lemma [8] (short LLL, also known as the Dobrushin condition [7] in statistical mechanics) and the explicit use of Shearer's measure [19] on $\mathbb{Z}_{(k)}$ 
to construct a series of probability measures dominating only trivial BPFs. Recall that Shearer's measure is the uniform minimal probability measure in the context of the LLL. It is also related to the grand canonical partition function of a lattice gas with both hard-core interaction and hard-core self-repulsion $[18,5]$.

Extending the work of Liggett, Schonmann \& Stacey in a natural way we demonstrate, that the use of Shearer's measure and the overall similarity between their proof and those concerning only Shearer's measure is not coincidence, but part of a larger picture. We show that there is a non-trivial uniform lower bound on the parameter vector of the BPF dominated by a BRF with marginal parameter vector $\vec{p}$ and dependency graph $G$ iff Shearer's measure with prescribed marginal parameter vector $\vec{p}$ exists on $G$.

After reparametrisation, the set of admissible vectors $\vec{p}$ is equivalent to the poly-disc of absolute and uniform convergence of the cluster expansion of the partition function of a hard-core lattice gas around fugacity $\overrightarrow{0}[18,5]$ allowing a high-temperature expansion [7]. This opens the door to a reinterpretation of results from cluster expansion techniques [12, 9, 4] or tree equivalence techniques [18, sections $6 \& 8]$, leading to improved estimates on admissible $\vec{p}$ for the domination problem. Possible future lines of research include the search for probabilistic interpretations of these combinatorial and analytic results.

The layout of this paper is as follows: we formulate the stochastic domination problem in section 2 and give a short introduction to Shearer's measure in section 3. Section 4 contains our new results, followed by examples of reinterpreted bounds in section 4.1. Finally section 6 deals with the weak invariant case and we refute the conjecture by Liggett, Schonmann \& Stacey concerning the minimality of Shearer's measure for the dominated parameter on $\mathbb{Z}_{(k)}$ in section 7.

\section{Setup and problem statement}

Let $G:=(V, E)$ be a locally finite graph. Denote by $\mathcal{N}(v)$ the set of neighbours of $v$ and by $\mathcal{N}_{1}(v):=\mathcal{N}(v) \uplus\{v\}$ the neighbourhood of $v$ including $v$ itself. For every $W \subseteq V$ denote by $G(W)$ the subgraph of $G$ induced by $W$.

Vectors are indexed by $V$, i.e. $\vec{x}:=\left(x_{v}\right)_{v \in V}$. Multiplication of vectors acts coordinate-wise. We have the natural partial order $\leq$ on real-valued vectors. Of particular importance is the notion of $\vec{x} \ll \vec{y}$, which means that there is a strict inequality in all coordinates. For $W \subseteq V$ let $\vec{x}_{W}:=\left(x_{v}\right)_{v \in W}$, where needed for disambiguation. We otherwise ignore superfluous coordinates. If we use a scalar $x$ in place of a vector $\vec{x}$ we mean to use $\vec{x}=x \overrightarrow{1}$ and call this the homogeneous setting. We always assume the relation $q=1-p$, also in vectorized form and when having corresponding subscripts. Denote by $\mathcal{X}_{V}:=\{0,1\}^{V}$ the compact space of binary configurations indexed by $V$. Equip $\mathcal{X}_{V}$ with the natural partial order induced by $\vec{x} \leq \vec{y}$ (isomorph to the partial order induced by the subset relation in $\mathcal{P}(V)$ ).

A Bernoulli random field (short BRF) $Y:=\left(Y_{v}\right)_{v \in V}$ on $G$ is a rv tak- 
ing values in $\mathcal{X}_{V}$, seen as a collection of Bernoulli rvs $Y_{v}$ indexed by $V$. A Bernoulli product field (short BPF) $X$ is a BRF where $\left(X_{v}\right)_{v \in V}$ is a collection of independent Bernoulli rvs. We write its law as $\Pi_{\vec{x}}^{V}$, where $x_{v}:=\Pi_{\vec{x}}^{V}\left(X_{v}=1\right)$.

A subset $A$ of the space $\mathcal{X}_{V}$ or the space $[0,1]^{V}$ is an up-set iff

$$
\forall \vec{x} \in A, \vec{y} \in \mathcal{X}_{V}: \quad \vec{x} \leq \vec{y} \Rightarrow \vec{y} \in A
$$

Replacing $\leq$ by $\geq$ in (1) we define a down-set.

We recall the definition of stochastic domination [14]. Let $Y$ and $Z$ be two BRFs on $G$. Denote by $\operatorname{Mon}(V)$ the set of monotone continuous functions from $\mathcal{X}_{V}$ to $\mathbb{R}$, that is $\vec{s} \leq \vec{t}$ implies $f(\vec{s}) \leq f(\vec{t})$. We say that $Y$ dominates $Z$ stochastically iff they respect monotonicity in expectation:

$$
Y \stackrel{s t}{\geq} Z \Leftrightarrow(\forall f \in \operatorname{Mon}(V): \quad \mathbb{E}[f(Y)] \geq \mathbb{E}[f(Z)]) .
$$

Equation (2) actually refers to the laws of $Y$ and $Z$. We abuse notation and treat a BRF and its law as interchangeable. Stochastic domination is equivalent to the existence of a coupling of $Y$ and $Z$ with $\mathbb{P}(Y \geq Z)=1$ [20].

The set of all dominated Bernoulli parameter vectors (short: set of dominated vectors) by a BRF $\mathrm{Y}$ is

$$
\Sigma(Y):=\left\{\vec{c}: Y \stackrel{s t}{\geq} \Pi_{\vec{c}}^{V}\right\}
$$

It describes all the different BPFs minorating $Y$ stochastically. The set $\Sigma(Y)$ is a closed down-set. The definition of dominated vector extends to a non-empty class $C$ of BRFs by

$$
\Sigma(C):=\bigcap_{Y \in C} \Sigma(Y)=\left\{\vec{c}: \forall Y \in C: Y \geq \Pi_{\vec{c}}^{\underline{V}}\right\} .
$$

For a class $C$ of BRFs denote by $C(\vec{p})$ the subclass consisting of BRFs with marginal parameter vector $\vec{p}$. We call a BPF with law $\Pi_{\vec{c}}^{V}$, respectively the vector $\vec{c}$, non-trivial iff $\vec{c} \gg 0$. Our main question is under which conditions all BRFs in a class $C$ dominate a non-trivial BPF. Even stronger, we ask whether they all dominate a common non-trivial BPF. Hence, given a class $C$, we investigate the set of parameter vectors guaranteeing non-trivial domination

$$
\mathcal{P}_{\text {dom }}^{C}:=\left\{\vec{p} \in[0,1]^{V}: \forall Y \in C(\vec{p}): \exists \vec{c} \gg \overrightarrow{0}: \vec{c} \in \Sigma(Y)\right\}
$$

and the set of parameter vectors guaranteeing uniform non-trivial domination

$$
\mathcal{P}_{\text {udom }}^{C}:=\left\{\vec{p} \in[0,1]^{V}: \exists \vec{c} \gg \overrightarrow{0}: \vec{c} \in \Sigma(C(\vec{p}))\right\} .
$$

We have the obvious inclusion

$$
\mathcal{P}_{\text {udom }}^{C} \subseteq \mathcal{P}_{\text {dom }}^{C}
$$


The main contribution of this paper is the characterization and description of certain properties of the sets (3d) and (3c) for some classes of BRFs.

A first class of BRFs is the so-called weak dependency class $[15,(1.1)]$ with marginal parameter $\vec{p}$ on $G$ :

$$
\mathcal{C}_{G}^{\text {weak }}(\vec{p}):=\left\{\operatorname{BRF} Y: \forall v \in V: \mathbb{P}\left(Y_{v}=1 \mid Y_{V \backslash \mathcal{N}_{1}(v)}\right) \geq p_{v}\right\} .
$$

In this context $G$ is a weak dependency graph of $Y$. We say that $G$ is a strong dependency graph of a $\mathrm{BRF} Y$ iff

$$
\forall W_{1}, W_{2} \subset V: \quad d\left(W_{1}, W_{2}\right)>1 \Rightarrow Y_{W_{1}} \text { is independent of } Y_{W_{2}} .
$$

In both cases, adding edges does not change $G$ 's status as dependency graph of $Y$. It is possible that $Y$ has multiple minimal dependency graphs [18, section 4.1]. The second class is the so-called strong dependency class [15, section 0] with marginal parameter $\vec{p}$ on $G$ :

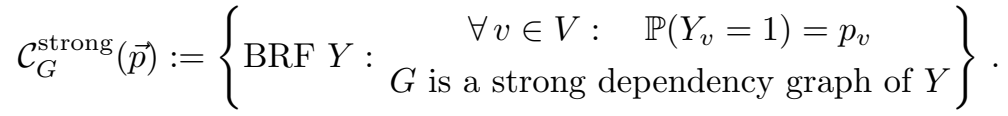

In particular

$$
\mathcal{C}_{G}^{\text {strong }}(\vec{p}) \subseteq \mathcal{C}_{G}^{\text {weak }}(\vec{p})
$$

In all but some trivial cases the inclusion 7 is strict (see after theorem 19).

\section{A primer on Shearer's measure}

This section contains an introduction to and overview of Shearer's measure. The following construction is due to Shearer [19]. Let $G:=(V, E)$ be finite and $\vec{p} \in[0,1]^{V}$. Recall that an independent set of vertices (in the graph theoretic sense) contains no adjacent vertices. Create a signed measure $\mu_{G, \vec{p}}$ on $\mathcal{X}_{V}$ with strong dependency graph $G$ by setting the marginals

$$
\forall W \subseteq V: \quad \mu_{G, \vec{p}}\left(Y_{W}=\overrightarrow{0}\right):= \begin{cases}\prod_{v \in W} q_{v} & W \text { independent } \\ 0 & W \text { not independent. }\end{cases}
$$

Use the inclusion-exclusion principle to complete $\mu_{G, \vec{p}}$ :

$$
\forall W \subseteq V: \quad \mu_{G, \vec{p}}\left(Y_{W}=\overrightarrow{0}, Y_{V \backslash W}=\overrightarrow{1}\right):=\sum_{\substack{W \subseteq T \subseteq V \\ T \text { indep }}}(-1)^{|T|-|W|} \prod_{v \in T} q_{v}
$$

Define the critical function of Shearer's signed measure on $G$ by

$$
\Xi_{G}: \quad[0,1]^{V} \rightarrow \mathbb{R} \quad \vec{p} \mapsto \Xi_{G}(\vec{p}):=\mu_{G, \vec{p}}\left(Y_{V}=\overrightarrow{1}\right)=\sum_{\substack{T \subseteq V \\ T \text { indep }}} \prod_{v \in T}\left(-q_{v}\right) .
$$

In graph theory (9) is also known as the independent set polynomial of $G$ [10, 13] and in lattice gas theory as the grand canonical partition function at negative 
fugacity $-\vec{q}[18$, section 2$]$. It satisfies a fundamental identity (an instance of a deletion-contraction identity)

$$
\forall v \in V, \vec{p} \in[0,1]^{V}: \quad \Xi_{G}(\vec{p})=\Xi_{G(V \backslash\{v\})}(\vec{p})-q_{v} \Xi_{G\left(V \backslash \mathcal{N}_{1}(v)\right)}(\vec{p}),
$$

derived from (9) by discriminating between independent sets containing $v$ and those which do not.

The set of admissible parameters for Shearer's measure is

$$
\begin{array}{rlrl}
\mathcal{P}_{s h}^{G}: & =\left\{\vec{p} \in[0,1]^{V}:\right. & & \left.\mu_{G, \vec{p}} \text { is a probability measure }\right\} \\
& =\left\{\vec{p} \in[0,1]^{V}:\right. & \left.\forall W \subseteq V: \quad \Xi_{G(W)}(\vec{p}) \geq 0\right\}
\end{array}
$$

The set $\mathcal{P}_{s h}^{G}$ is closed, strictly decreasing when adding edges and an up-set [18, proposition $2.15(\mathrm{~b})$ ], hence connected. It always contains the vector $\overrightarrow{1}$ and, unless $E=\emptyset$, never the vector $\overrightarrow{0}$. Therefore it is a non-trivial subset of $[0,1]^{V}$ (see also section 4.1). The function $\Xi_{G}$ is strictly increasing on $\mathcal{P}_{s h}^{G}$. It is convenient to subdivide $\mathcal{P}_{s h}^{G}$ further into its boundary

$$
\partial \mathcal{P}_{s h}^{G}:=\left\{\vec{p}: \Xi_{G}(\vec{p})=0 \text { and } \mu_{G, \vec{p}} \text { is a probability measure }\right\}
$$

and interior (both seen as subsets of the space $[0,1]^{V}$ )

$$
\begin{aligned}
\stackrel{\circ}{\mathcal{P}}_{s h}^{G}:=\mathcal{P}_{s h}^{G} \backslash \partial \mathcal{P}_{s h}^{G} & =\left\{\vec{p}: \Xi_{G}(\vec{p})>0 \text { and } \mu_{G, \vec{p}} \text { is a probability measure }\right\} \\
& =\left\{\vec{p}: \Xi_{H}(\vec{p})>0 \text { for all subgraphs } H \text { of } G\right\} .
\end{aligned}
$$

Finally we see that for $\vec{p} \in \mathcal{P}_{s h}^{G}$ the probability measure $\mu_{G, \vec{p}}$

$$
\text { has dependency graph } G \text {, }
$$

has marginal parameter $\vec{p}$, i.e $\forall v \in V: \mu_{G, \vec{p}}\left(Y_{v}=1\right)=p_{v}$,

and forbids neighbouring 0 s, i.e. $\forall(v, w) \in E: \mu_{G, \vec{p}}\left(Y_{v}=Y_{w}=0\right)=0$.

Properties (14a) and (14b) are equivalent to $\mu_{G, \vec{p}} \in \mathcal{C}_{G}^{\text {strong }}(\vec{p})$. Every probability measure $\nu$ on $\mathcal{X}_{V}$ fulfilling (14) can be constructed by (8) and thus coincides with $\mu_{G, \vec{p}}$. Hence (14) characterizes $\mu_{G, \vec{p}}$.

The importance of Shearer's measure is due to its uniform minimality with respect to certain conditional probabilities:

Lemma 1 ([19, theorem 1]). Let $\vec{p} \in \mathcal{P}_{\text {sh }}^{G}$ and $Z \in \mathcal{C}_{G}^{\text {weak }}(\vec{p})$. Then $\forall W \subseteq V$ :

$$
\mathbb{P}\left(Z_{W}=\overrightarrow{1}\right) \geq \mu_{G, \vec{p}}\left(Y_{W}=\overrightarrow{1}\right)=\Xi_{G(W)}(\vec{p}) \geq 0
$$

and $\forall W \subseteq U \subseteq V:$ if $\Xi_{G(W)}(\vec{p})>0$, then

$$
\mathbb{P}\left(Z_{U}=\overrightarrow{1} \mid Z_{W}=\overrightarrow{1}\right) \geq \mu_{G, \vec{p}}\left(Y_{U}=\overrightarrow{1} \mid Y_{W}=\overrightarrow{1}\right)=\frac{\Xi_{G(U)}(\vec{p})}{\Xi_{G(W)}(\vec{p})} \geq 0 .
$$

It is the cost of isolating $0 \mathrm{~s}$, that drives and is equivalent to the above minimality. 
If $G$ is infinite define

$$
\mathcal{P}_{s h}^{G}:=\bigcap_{E^{\prime} \subseteq E,\left|E^{\prime}\right|<\infty} \mathcal{P}_{s h}^{\left(V, E^{\prime}\right)} \quad \text { and } \quad \stackrel{\circ}{\mathcal{P}}_{s h}^{G}:=\bigcap_{E^{\prime} \subseteq E,\left|E^{\prime}\right|<\infty} \stackrel{\circ}{\mathcal{P}}_{s h}^{\left(V, E^{\prime}\right)} .
$$

This is well defined $[18,(8.4)]$. The set $\stackrel{\circ}{\mathcal{P}}_{s h}^{G}$ is not the interior of the closed set $\mathcal{P}_{s h}^{G}$ (discussed in detail in [18, theorem 8.1]). For $\vec{p} \in \mathcal{P}_{s h}^{G}$ the family of marginals $\left\{\mu_{G(W), p}: W \subsetneq V, W\right.$ finite $\}$ forms a consistent family à la Kolmogorov [3, (36.1) \& (36.2)]. Hence Kolmogorov's existence theorem [3, theorem 36.2] establishes the existence of an extension of this family, which we call $\mu_{G, \vec{p}}$. The $\pi-\lambda$ theorem [3, theorem 3.3] asserts the uniqueness of this extension. Furthermore $\mu_{G, \vec{p}}$ has all the properties listed in (14) on the infinite graph $G$. Conversely let $\nu$ be a probability measure having the properties (14). Then all its finite marginals have them, too, and they coincide with Shearer's measure. Hence by the uniqueness of the Kolmogorov extension $\nu$ coincides with $\mu_{G, \vec{p}}$ and (14) characterizes $\mu_{G, \vec{p}}$ also on infinite graphs.

\section{Main results and discussion}

Our main result is

Theorem 2. For every locally finite graph $G$, we have

$$
\mathcal{P}_{\text {dom }}^{\mathcal{C}_{G}^{\text {weak }}}=\mathcal{P}_{\text {udom }}^{\mathcal{C}_{G}^{\text {weak }}}=\mathcal{P}_{\text {dom }}^{\mathcal{C}_{G}^{\text {strong }}}=\mathcal{P}_{\text {udom }}^{\mathcal{C}_{G}^{\text {strong }}}=\stackrel{\circ}{\mathcal{P}}_{\text {sh }}^{G} .
$$

Its proof is in section 5 . Theorem 2 consists of two a priori unrelated statements: The first one consists of the left three equalities in (17): uniform and non-uniform domination of a non-trivial BPF are the same, and even taking the smaller class $\mathcal{C}_{G}^{\text {strong }}$ does not admit more $\vec{p}$. The second one is that these sets are equivalent to the set of parameters for which Shearer's measure exists. The minimality of Shearer's measure (see lemma 1) lets us construct BRFs dominating only trivial BPFs for $\vec{p} \notin \stackrel{\circ}{\mathcal{P}}_{\text {sh }}^{G}$ (see section 5.2) and clarifies the role Shearer's measure played as a counterexample in the work of Liggett, Schonmann \& Stacey [15, section 2]. Even more, this minimality implies an explicit lower bound for the non-trivial uniform dominated vector:

Theorem 3. For $\vec{p} \in \stackrel{\circ}{\mathcal{P}}_{\text {sh }}^{G}$, define the vector $\vec{c}$ component-wise by

$$
c_{v}:= \begin{cases}1 & \text { if } p_{v}=1 \\ 1-\left(1-\Xi_{G_{v}}(\vec{p})\right)^{1 /\left|V_{v}\right|} & \text { if } p_{v}<1 \text { and }\left|V_{v}\right|<\infty \\ q_{v} \min \left\{q_{w}: w \in \mathcal{N}(v) \cap V_{v}\right\} & \text { if } p_{v}<1 \text { and }\left|V_{v}\right|=\infty,\end{cases}
$$

where $V_{v}$ are the vertices of the connected component of $v$ in the subgraph of $G$ induced by all vertices $v$ with $p_{v}<1$. Then $\overrightarrow{0} \ll \vec{c} \in \Sigma\left(\mathcal{C}_{G}^{\text {weak }}(\vec{p})\right)$.

The proof of theorem 3 is in section 5.4. For infinite, connected $G$ we have a discontinuous transition in $\vec{c}$ as $\vec{p}$ approaches the boundary of $\mathcal{P}_{s h}^{G}$ (18c), while in the finite case it is continuous $(18 \mathrm{~b})$. On the other hand there are classes of BRFs having a continuous transition also in the infinite case, for example the class of 2-factors on $\mathbb{Z}$ [15, theorem 3.0]. 
Our proof trades accuracy in capturing all of $\stackrel{\circ}{\mathcal{P}}_{s h}^{G}$ against accuracy in the lower bound for the parameter of the dominated BPF. Intuitively it is clear, that $\Sigma\left(\mathcal{C}_{G}^{\text {weak }}(\vec{p})\right)$ should increase with $\vec{p}(29)$, but our explicit lower bound (18c) decreases in $\vec{p}$. There is an explicit growing lower bound already shown by Liggett, Schonmann \& Stacey [15, corollary 1.4], although only on a restricted set of parameters (19).

Equation (15a) does not imply, that $\mu_{G, \vec{p}} \stackrel{s t}{\leq} Y$ for all $Y \in \mathcal{C}_{G}^{\text {weak }}(\vec{p})$ : for a finite $W \subsetneq V$ take $f:=1-\mathbb{I}_{\{\overrightarrow{0}\}} \in \operatorname{Mon}(W)$ and see that $\Pi_{\vec{p}}^{W} \stackrel{s t}{\geq} \mu_{G(W), \vec{p}}$. Furthermore $\Sigma\left(\mu_{G, \vec{p}}\right)$ is neither minimal nor maximal (with respect to set inclusion) in the class $\mathcal{C}_{G}^{\text {weak }}(\vec{p})$. The maximal law is $\Pi_{\vec{p}}^{W}$ itself, as $[\overrightarrow{0}, \vec{p}]=\Sigma\left(\Pi_{\vec{p}}^{W}\right)$. We give a counterexample to the minimality of $\Sigma\left(\mu_{G, \vec{p}}\right)$ in section 7 .

\subsection{Reinterpretation of bounds}

Theorem 2 allows the application of conditions for admissible $\vec{p}$ for $\mathcal{P}_{u d o m}^{\mathcal{C}_{G}^{\text {weak }}}$ to $\stackrel{\circ}{\mathcal{P}}_{s h}^{G}$ and vice-versa. Hence we can play questions about the existence of a BRF dominating only trivial BPFs or the existence of Shearer's measure back and forth. In the following we list known necessary or sufficient conditions for $\vec{p}$ to lie in $\stackrel{\circ}{\mathcal{P}}_{s h}^{G}$, most of them previously unknown for the domination problem. We assume that $G$ contains no isolated vertices. The classical sufficient condition for the existence of Shearer's measure has been established independently several times and is known as either the "Lovász Local Lemma" [8] in graph theory or the "Dobrushin condition" [7, theorem 6.1] in statistical mechanics:

Theorem 4 (version of $[9,(2.13)])$. Let $\vec{p} \in[0,1]^{V}$. If there exists $\left.\vec{s} \in\right] 0, \infty\left[{ }^{V}\right.$ such that

$$
\forall v \in V: \quad q_{v} \prod_{w \in \mathcal{N}_{1}(v)}\left(1+s_{w}\right) \leq s_{v}
$$

then $\vec{p} \in \stackrel{\circ}{\mathcal{P}}_{\text {sh }}^{G}$.

In the homogeneous case there has been again a parallel and independent improvement on theorem 4 by Liggett, Schonmann \& Stacey in probability theory and Scott \& Sokal in statistical mechanics. Here $p_{s h}^{G}$ is identified with the endpoint of the interval $\left[p_{s h}^{G}, 1\right]$ corresponding to $\stackrel{\circ}{\mathcal{P}}_{s h}^{G}$.

Theorem 5 ([15, theorem 1.3], [18, corollary 5.7]). If $G$ is uniformly bounded with degree $D$, then

$$
p_{s h}^{G} \leq 1-\frac{(D-1)^{(D-1)}}{D^{D}} .
$$

This leads to the only two cases of infinite graphs where $p_{s h}^{G}$ is exactly known, namely the $D$-regular tree $\mathbb{T}_{D}$ with $p_{s h}^{\mathbb{T}_{D}}=1-\frac{(D-1)^{(D-1)}}{D^{D}}$ and $\mathbb{Z}_{(k)}$, the $k$-fuzz of $\mathbb{Z}$, with $p_{s h}^{\mathbb{Z}_{(k)}}=1-\frac{k^{k}}{(k+1)^{(k+1)}}$. The complementary inequality is $[19$, before theorem 2] and [15, corollary 2.2] for $\mathbb{T}_{d}$ and $\mathbb{Z}_{(k)}$ respectively. In these cases explicit constructions of Shearer's measure are possible. See for example the construction as a $(k+1)$-factor in the case of $\mathbb{Z}_{(k)}[16$, section 4.2$]$.

Fernández \& Procacci derived another more recent and elaborate sufficient condition for a vector $\vec{p}$ to lie in $\stackrel{\circ}{\mathcal{P}}_{s h}^{G}$ : 
Theorem 6 ([9, theorem 1$])$. Let $\vec{p} \in[0,1]^{V}$. If there exists $\left.\vec{s} \in\right] 0, \infty\left[{ }^{V}\right.$, such that

$$
\forall v \in V: \quad q_{v} \Xi_{G\left(\mathcal{N}_{1}(v)\right)}(-\vec{s}) \leq s_{v}
$$

then $\vec{p} \in \stackrel{\circ}{\mathcal{P}}_{\text {sh }}^{G}$.

The minus in (21) stems from their cluster expansion technique and assures that $\Xi_{G\left(\mathcal{N}_{1}(v)\right)}(-\vec{s}) \geq 1$, whence $q_{v} \leq 1$. The condition takes into account the local structure of $G$, via the triangles in $\mathcal{N}_{1}(v)$. It thus improves upon the LLL, which only considers the degree of $v$.

We present an example of a necessary condition by Scott \& Sokal in the homogeneous case. Define the upper growth rate of a tree $\mathbb{T}$ rooted at $o$ by

$$
\overline{\operatorname{gr}}(\mathbb{T}):=\limsup _{n \rightarrow \infty}\left|V_{n}\right|^{1 / n},
$$

where $V_{n}$ are the vertices of $\mathbb{T}$ at distance $n$ from $o$. Then we have

Theorem 7 ([18, proposition 8.3]). Let $G$ be infinite. Then

$$
p_{s h}^{G} \geq 1-\frac{\overline{\operatorname{gr}}(\mathbb{T})^{\overline{\operatorname{gr}(\mathbb{T})}}}{(\overline{\operatorname{gr}}(\mathbb{T})+1)^{(\overline{\operatorname{gr}}(\mathbb{T})+1)}}
$$

Here $\mathbb{T}$ is a particular pruned subtree of the $S A W$ (self-avoiding-walk) tree of $G$ defined in [18, section 6.2].

The pruned subtree $\mathbb{T}$ referred to above stems from a recursive expansion of the critical function via the fundamental identity (10) and the subsequent identification of this calculation with the one on $\mathbb{T}$. It is a subtree of the SAW tree of $G$, which not only avoids revisiting previously visited nodes, but also some of their neighbours. An example demonstrating this result is the following statement $[18,(8.53)]$ :

$$
p_{s h}^{\mathbb{Z}^{d}} \geq 1-\frac{d^{d}}{(d+1)^{(d+1)}} .
$$

It follows from the fact that one can embed a regular rank $d$ rooted tree in the pruned SAW $\mathbb{T}$ of $\mathbb{Z}^{d}$, whence $d \leq \overline{\operatorname{gr}}(\mathbb{T})$. For the full details we refer the reader to $[18$, sections $6 \& 8]$.

\section{Proofs}

We prove theorem 2 by showing all inclusions outlined in figure 1 . The four center inclusions follow straight from (3e) and (7). The core part are two inclusions marked (UD) and (ND) in figure 1. The second inclusion (ND) generalizes an idea of Liggett, Schonmann \& Stacey in section 5.2. The key is the usage of Shearer's measure on finite subgraphs $H$ for suitable $\vec{p} \in \partial \mathcal{P}_{s h}^{H}$ to create BRFs dominating only trivial BPFs. Our novel contribution is the inclusion (UD). It replaces the LLL style proof for restricted parameters employed in [15, proposition 1.2] by an optimal bound reminiscent of the optimal bound presented in [18, section 5.3], using the fundamental identity (10) to full extent. After some preliminary work on Shearer's measure in section 5.3 we prove the inclusion (UD) in section 5.4. 


$$
\begin{aligned}
& \stackrel{\circ}{\mathcal{P}}_{\text {sh }}^{G} \stackrel{\text { (UD) }}{\subseteq} \mathcal{P}_{\text {udom }}^{\mathcal{C}_{G}^{\text {weak }}} \subseteq \mathcal{P}_{\text {dom }}^{\mathcal{C}_{G}^{\text {weak }}} \\
& \text { in in } \\
& \mathcal{P}_{\text {udom }}^{\mathcal{C}_{G}^{\text {strong }}} \subseteq \mathcal{P}_{\text {dom }}^{\mathcal{C}_{G}^{\text {strong }}} \stackrel{(\mathrm{ND})}{\subseteq} \stackrel{\circ}{\mathcal{P}}_{\text {sh }}^{G}
\end{aligned}
$$

Figure 1: Inclusions in the proof of (17).

\subsection{Tools for stochastic domination}

In this section we list useful statements related to stochastic domination between BRFs.

Lemma 8 ([14, chapter II, page 79]). Let $Y, Z$ be two BRFs indexed by $V$, then

$$
Y \stackrel{s t}{\geq} Z \quad \Leftrightarrow \quad\left(\forall \text { finite } W \subseteq V: Y_{W} \stackrel{s t}{\geq} Z_{W}\right)
$$

We build on the following technical result, inspired by [17, lemma 1].

Proposition 9. If $Z:=\left\{Z_{n}\right\}_{n \in \mathbb{N}}$ is a BRF with

$$
\forall n \in \mathbb{N}, \vec{s}_{[n]} \in \mathcal{X}_{[n]}: \quad \mathbb{P}\left(Z_{n+1}=1 \mid Z_{[n]}=\vec{s}_{[n]}\right) \geq p_{n}
$$

then $Z \stackrel{s t}{\geq} \Pi_{\vec{p}}^{\mathbb{N}}$.

Proof. Essentially the same inductive proof as in [17, lemma 1].

If $Y$ and $Z$ are two independent BRFs with marginal vectors $\vec{p}$ and $\vec{r}$, then we denote by

$$
Y \wedge Z:=\left(Y_{v} \wedge Z_{v}\right)_{v \in V}
$$

the vertex-wise minimum with marginal vector $\vec{p} \vec{r}$. Coupling shows that for every two BRFs $Y$ and $Z$ we have

$$
Y \wedge Z \stackrel{s t}{\leq} Y
$$

and if $X$ is a third $\mathrm{BRF}$ independent of $(Y, Z)$ also

$$
Y \stackrel{s t}{\geq} Z \Rightarrow(Y \wedge X) \stackrel{s t}{\geq}(Z \wedge X)
$$

Proposition 10. For each dependency class $C$ used in this paper and all $\vec{p}$ and $\vec{r}$, we have

$$
\Sigma(C(\vec{p} \vec{r})) \subseteq \Sigma(C(\vec{p})) .
$$

Proof. Let $\vec{c} \in \Sigma(C(\vec{p} \vec{r}))$. Let $Y \in C(\vec{p})$ and $X$ be $\Pi_{\vec{r}}^{V}$-distributed independently of $Y$. Using (28) we get $\Pi_{\vec{c}}^{V} \stackrel{s t}{\leq} Y \wedge X \stackrel{s t}{\leq} Y$, whence $\vec{c} \in \Sigma(Y)$. As this holds for every $Y \in C(\vec{p})$ we have $\vec{c} \in \Sigma(C(\vec{p}))$. 


\subsection{Nondomination}

In this section we prove inclusion (ND) from figure 1 , that is $\mathcal{P}_{d o m}^{\mathcal{C}_{G}^{\text {strong }}} \subseteq \stackrel{\circ}{\mathcal{P}}_{s h}^{G}$. The plan is as follows: in lemma 11 we recall a coupling involving Shearer's measure on a finite graph $H$ [19, proof of theorem 1], which creates a BRF dominating only trivial BPFs for every $\vec{p} \notin \stackrel{\circ}{\mathcal{P}}{ }_{s h}^{H}$. In proposition 12 we generalize an approach used by Liggett, Schonmann \& Stacey [15, theorem 2.1] to arbitrary graphs and inhomogeneous parameters. For infinite $G$ and $\vec{p} \notin \stackrel{\circ}{\mathcal{P}}_{s h}^{G}$, we find a suitable finite subgraph $H$ of $G$ on which to effectuate the above mentioned coupling and extend it with an independent BPF on the complement. The resulting BRF dominates only trivial BPFs.

Lemma 11 ([19, proof of theorem 1]). Let $G$ be finite. If $\vec{p} \notin \stackrel{\circ}{\mathcal{P}}_{\text {sh }}^{G}$, then there exists a BRF $Z \in \mathcal{C}_{G}^{\text {strong }}(\vec{p})$ with $\mathbb{P}\left(Z_{V}=\overrightarrow{1}\right)=0$.

Proof. As $\vec{p} \notin \stackrel{\circ}{\mathcal{P}}_{s h}^{G}$ and $\overrightarrow{1} \in \stackrel{\circ}{\mathcal{P}}_{\text {sh }}^{G}$ the line segment $[\vec{p}, \overrightarrow{1}]$ crosses $\partial \mathcal{P}_{s h}^{G}$ at the vector $\vec{r}$ (unique because $\stackrel{\circ}{\mathcal{P}}_{s h}^{G}$ is an up-set [18, proposition 2.15 (b)]). Let $\vec{x}$ be the solution of $\vec{p}=\vec{x} \vec{r}$. Let $Y$ be $\mu_{G, \vec{r}}$-distributed and $X$ be $\Pi_{\vec{x}}^{V}$-distributed independently of $Y$. Set $Z:=Y \wedge X$. Then $Z \in \mathcal{C}_{G}^{\text {strong }}(\vec{p})$ and

$$
\mathbb{P}\left(Z_{V}=\overrightarrow{1}\right)=\mathbb{P}\left(X_{V}=\overrightarrow{1}\right) \mu_{G, \vec{r}}\left(Y_{V}=\overrightarrow{1}\right)=0 .
$$

Proposition 12. We have $\mathcal{P}_{\text {dom }}^{\mathcal{C}_{G}^{\text {strong }}} \subseteq \stackrel{\circ}{\mathcal{P}}_{\text {sh }}^{G}$.

Proof. Let $\vec{p} \notin \stackrel{\circ}{\mathcal{P}}_{s h}^{G}$. Then there exists a finite set $W \subseteq V$ with $\vec{p}_{W} \notin \stackrel{\circ}{\mathcal{P}}_{s h}^{G(W)}$. Using lemma 11 create a $Y_{W} \in \mathcal{C}_{G(W)}^{\text {strong }}(\vec{p})$ with $\mathbb{P}\left(Y_{W}=\overrightarrow{1}\right)=0$. Extend this to a $Y \in \mathcal{C}_{G}^{\text {strong }}(\vec{p})$ by letting $Y_{V \backslash W}$ be $\Pi_{\vec{p}_{V \backslash W}}^{V \backslash W}$-distributed independently of $Y_{W}$.

Suppose that $Y \stackrel{s t}{\geq} X$, where $X$ is $\Pi_{\vec{x}}^{V}$-distributed. Then lemma 8 implies that $Y_{W} \stackrel{s t}{\geq} X_{W}$ and, using $f:=\mathbb{I}_{\{\overrightarrow{1}\}} \in \operatorname{Mon}(W)$, that

$$
0=\mathbb{P}\left(Y_{W}=\overrightarrow{1}\right)=\mathbb{E}\left[f\left(Y_{W}\right)\right] \geq \mathbb{E}\left[f\left(X_{W}\right)\right]=\mathbb{P}\left(X_{W}=\overrightarrow{1}\right)=\prod_{v \in W} x_{v} \geq 0 .
$$

Hence there exists a $v \in W$ with $x_{v}=0$, whence $\vec{x} \ngtr \overrightarrow{0}$ and $\vec{p} \notin \mathcal{P}_{d o m}^{\mathcal{C}_{G}^{\text {strong }}}$.

\subsection{One vertex open extension probabilities}

In this section we reencode our knowledge of Shearer's measure from the critical functions as ratios of critical functions, that is conditional probabilities of the form "open on some vertices | open on some other vertices". These are exactly the ones Shearer's measure is minimal for $(15 \mathrm{~b})$. This viewpoint admits a more succinct formulation of the fundamental identity (10) and bounds in proposition 14. The notion of "escaping" pair introduced in this section is inspired by [19, theorem 2]. It allows us to push the mass of unwanted conditional events away. We obtain lower bounds on conditional events of the above form, which are independent of size of the condition, as long as the escape persists. 
For finite $W \subseteq V$ with $v \notin W$ and when $\Xi_{G(W)}(\vec{p})>0$ define the one vertex open extension probability of $(W, v)$ by

$$
\alpha_{W}^{v}(\vec{p}):=\mu_{G, \vec{p}}\left(Y_{v}=1 \mid Y_{W}=\overrightarrow{1}\right) .
$$

Reformulate the fundamental identity (10) as

$$
\alpha_{W}^{v}(\vec{p})=1-\frac{q_{v}}{\prod_{i=1}^{m} \alpha_{W \backslash\left\{w_{i}, \ldots, w_{m}\right\}}^{w_{i}}(\vec{p})},
$$

where $W \cap \mathcal{N}(v)=:\left\{w_{1}, \ldots, w_{m}\right\}$.

Definition 13. Call the pair $(W, v)$, respectively $\alpha_{W}^{v}$, escaping iff $\mathcal{N}(v) \backslash W \neq \emptyset$ and call every vertex $w \in \mathcal{N}(v) \backslash W$ an escape of $(W, v)$.

Proposition 14. Let $\vec{p} \in \mathcal{P}_{s h}^{G}$, then

$$
\forall(W, v): \quad \alpha_{W}^{v}(\vec{p}) \leq p_{v}
$$

and

$$
\forall(W, v), w \in \mathcal{N}(v) \backslash W: \quad q_{w} \leq \alpha_{W}^{v}(\vec{p}) .
$$

Proof. We use the fundamental identity (31) to see that

$$
\alpha_{W}^{v}(\vec{p})=1-\frac{q_{v}}{\prod \alpha_{\star}^{\star}(\vec{p})} \leq 1-q_{v}=p_{v} .
$$

Likewise, if $(W, v)$ is escaping with escape $w \in \mathcal{N}(v) \backslash W$, then (31) yields

$$
0 \leq \alpha_{W \uplus\{v\}}^{w}(\vec{p})=1-\frac{q_{w}}{\alpha_{W}^{v}(\vec{p}) \prod \alpha_{\star}^{\star}(\vec{p})} \leq 1-\frac{q_{w}}{\alpha_{W}^{v}(\vec{p})}
$$

hence $q_{w} \leq \alpha_{W}^{v}(\vec{p})$.

Proposition 15. Let $\vec{p} \in \mathcal{P}_{s h}^{G}$. Then $\alpha_{W}^{v}(\vec{p})$ decreases, as $W$ increases.

Proof. If $p_{v}=0$, then $\alpha_{W}^{v}(\vec{p})=0$ for all $W$. If $p_{w}=0$ for $w \in W$ and $v$ connected to $w$ then $\alpha_{W}^{v}(\vec{p})$ is not defined. Hence for the remainder of this proof assume $\overrightarrow{0} \ll \vec{p}$. We prove the statement by simultaneous induction for all $v$ over the cardinality of $W$. The base case is

$$
\alpha_{\emptyset}^{v}(\vec{p})=1-q_{v} \begin{cases}\geq \frac{1-q_{v}-q_{w}}{1-q_{w}}=\alpha_{\{w\}}^{v}(\vec{p}) & \text { if } v \backsim w \\ =1-q_{v}=\alpha_{\{w\}}^{v}(\vec{p}) & \text { if } v \nmid w .\end{cases}
$$

For the induction step we add just one vertex $w$ to $W$ and set $U:=W \uplus\{w\}$. Let $\left\{w_{1}, \ldots, w_{m}\right\}:=\mathcal{N}(v) \cap U$. First assume that $w \nmid v$. Using the fundamental identity (31) we have

$$
\alpha_{U}^{v}(\vec{p})=1-\frac{q_{v}}{\prod_{i=1}^{m} \alpha_{U \backslash\left\{w_{i}, \ldots, w_{m}\right\}}^{w_{i}}(\vec{p})} \leq 1-\frac{q_{v}}{\prod_{i=1}^{m} \alpha_{W \backslash\left\{w_{i}, \ldots, w_{m}\right\}}^{w_{i}}(\vec{p})}=\alpha_{W}^{v}(\vec{p}) .
$$

Secondly assume that $v \backsim w=w_{m}$. Hence

$\alpha_{U}^{v}(\vec{p})=1-\frac{q_{v}}{\prod_{i=1}^{m} \alpha_{U \backslash\left\{w_{i}, \ldots, w_{m}\right\}}^{w_{i}}(\vec{p})} \leq 1-\frac{q_{v}}{\prod_{i=1}^{m-1} \alpha_{W \backslash\left\{w_{i}, \ldots, w_{m-1}\right\}}^{w_{i}}(\vec{p})}=\alpha_{W}^{v}(\vec{p})$. 


\subsection{Domination}

In this section we prove inclusion (UD) from figure 1 , that is $\stackrel{\circ}{\mathcal{P}}_{\text {sh }}^{G} \subseteq \mathcal{P}_{\text {udom }}^{\mathcal{C}_{G}^{\text {weak }}}$. We split the proof in two and deal with finite and infinite $G$ separately in proposition 16 and 17, respectively. Additionally (33) and (34) combined yield a proof of (18) from theorem 3.

On a finite graph our approach is direct: proposition 16 uses the minimality of $\mu_{G, \vec{p}}$ to construct a homogeneous nontrivial dominated vector $\overrightarrow{0} \ll \vec{c} \in$ $\Sigma\left(\mathcal{C}_{G}^{\text {weak }}(\vec{p})\right)$. For an infinite graph the situation is more involved and we use a technique of Antal \& Pisztora [2, pages 1040-1041]: Suppose you have a $Y \in \mathcal{C}_{G}^{\text {weak }}(\vec{p})$ with $\overrightarrow{0} \ll \vec{y} \in \Sigma(Y)$. Let $X$ be $\Pi_{V}^{\vec{x}}$ with $\overrightarrow{0} \ll \vec{x}$ independently of $Y$ and set $Z:=X \wedge Y$. Then $\overrightarrow{0} \ll \vec{x} \vec{y} \in \Sigma(Z) \subseteq \Sigma(Y)$, that is an independent non-trivial i.i.d. perturbation does not change the quality of $Y$ 's domination behaviour.

Proposition 18 uses this perturbation to blame adjacent 0 realizations of $Z$ on $X$ instead of $Y$, leading to the uniform technical minorization (35):

$$
\mathbb{P}\left(Z_{v}=1 \mid Z_{W}=\vec{s}_{W}\right) \geq q_{v} \alpha_{W}^{v}(\vec{p}),
$$

connecting the domination problem with Shearer's measure. Finally in proposition 17 we ensure to look at only escaping $(W, v) \mathrm{s}$, hence getting rid of the $\alpha_{W}^{v}(\vec{p})$ term. This allows us to apply proposition 9 and guarantee stochastic domination of a non-trivial BPF.

Proposition 16. Let $G$ be finite and $\vec{p} \in \stackrel{\circ}{\mathcal{P}}_{\text {sh }}^{G}$. Let $X$ be $\Pi_{c}^{V}$-distributed with

$$
c:=1-\left(1-\Xi_{G}(\vec{p})\right)^{1 /|V|}>0 .
$$

Then every $Y \in \mathcal{C}_{G}^{\text {weak }}(\vec{p})$ fulfils $Y \stackrel{s t}{\geq} X$, hence $\vec{p} \in \mathcal{P}_{\text {udom }}^{\mathcal{C}_{G}^{\text {weak }}}$.

Proof. The choice of $\vec{p}$ implies that $\Xi_{G}(\vec{p})>0$, therefore $c>0$, too. Let $f \in \operatorname{Mon}(V)$ and $Y \in \mathcal{C}_{G}^{\text {weak }}(\vec{p})$. Then

$$
\begin{aligned}
& \mathbb{E}[f(X)] \\
= & \sum_{\vec{s} \in \mathcal{X}_{V}} f(\vec{s}) \mathbb{P}(X=\vec{s}) \\
\leq & f(\overrightarrow{0}) \mathbb{P}(X=\overrightarrow{0})+f(\overrightarrow{1}) \mathbb{P}(X \neq \overrightarrow{0}) \\
= & f(\overrightarrow{0})(1-c)^{|V|}+f(\overrightarrow{1})\left[1-(1-c)^{|V|}\right] \\
= & f(\overrightarrow{0})\left[1-\Xi_{G}(\vec{p})\right]+f(\overrightarrow{1}) \Xi_{G}(\vec{p}) \\
\leq & f(\overrightarrow{0}) \mathbb{P}(Y \neq \overrightarrow{1})+f(\overrightarrow{1}) \mathbb{P}(Y=\overrightarrow{1}) \\
\leq & \sum_{\vec{s} \in \mathcal{X}_{V}} f(\vec{s}) \mathbb{P}(Y=\vec{s}) \\
= & \mathbb{E}[f(Y)] .
\end{aligned}
$$$$
\text { minimality of Shearer's measure (15a) }
$$

monotonicity of $f$

Hence $X \stackrel{s t}{\leq} Y$. As $\overrightarrow{0} \ll c \overrightarrow{1}$ we have $\vec{p} \in \mathcal{P}_{\text {udom }}^{\mathcal{C}_{G}^{\text {weak }}}$. 
Proposition 17. Let $G$ be infinite and connected. Let $\overrightarrow{1} \gg \vec{p} \in \stackrel{\circ}{\mathcal{P}}_{\text {sh }}^{G}$. Define the vector $\vec{c}$ by

$$
\forall v \in V: \quad c_{v}:=q_{v} \min \left\{q_{w}: w \in \mathcal{N}(v)\right\}
$$

Then $\vec{c} \gg \overrightarrow{0}$ and every $Y \in \mathcal{C}_{G}^{\text {weak }}(\vec{p})$ fulfils $Y \geq \Pi_{\vec{c}}^{V}$, whence $\vec{p} \in \mathcal{P}_{\text {udom }}^{\mathcal{C}_{G}^{\text {weak }}}$.

Remark. Proposition 17 motivated the definition of "escaping" pairs: it allows for non-trivial lower bounds for escaping $\alpha_{W}^{v}(\vec{p})$, in a correctly chosen ordering of a finite subgraph. Arbitrary $\alpha_{W}^{v}(\vec{p})$ defy control at the boundary of $\stackrel{\circ}{\mathcal{P}}_{s h}^{G}$.

Proof. We show, that $Y_{W} \stackrel{s t}{\geq} \Pi_{\vec{c}_{W}}^{W}$, for every finite $W \subsetneq V$. Admitting this momentarily, lemma 8 asserts that $Y \geq \Pi_{\vec{c}}^{V t}$. Conclude as $\vec{p} \ll \overrightarrow{1}$ implies, that $\vec{c} \gg \overrightarrow{0}$.

Choose a finite $W \subsetneq V$ and let $|W|=: n$. As $G$ is connected and infinite, there is a vertex $v_{n} \in W$ which has a neighbour $w_{n}$ in $V \backslash W$. It follows, that $\left(W \backslash\left\{v_{n}\right\}, v_{n}\right)$ is escaping with escape $w_{n} \in \mathcal{N}\left(v_{n}\right) \backslash W$. Apply this argument recursively to $W \backslash\left\{v_{n}\right\}$ and thus produce a total ordering $v_{1} \prec \ldots \prec v_{n}$ of $W$, where, setting $W_{i}:=\left\{v_{1}, \ldots, v_{i-1}\right\}$, every $\left(W_{i}, v_{i}\right)$ is escaping with escape $w_{i} \in \mathcal{N}\left(v_{i}\right) \backslash W_{i}$.

Let $X$ be $\Pi_{\vec{q}}^{V}$-distributed independently of $Y$. Set $Z:=Y \wedge X$. Then (35) from proposition 18 and the minoration for escaping pairs (32b) combine to

$$
\forall i \in[n], \forall \vec{s}_{W_{i}} \in \mathcal{X}_{W_{i}}: \quad \mathbb{P}\left(Z_{v_{i}}=1 \mid Z_{W_{i}}=\vec{s}_{W_{i}}\right) \geq \alpha_{W_{i}}^{v_{i}}(\vec{p}) q_{v_{i}} \geq q_{w_{i}} q_{v_{i}} \geq c_{v_{i}} .
$$

This is sufficient for proposition 9 to construct a coupling with $Z_{W} \stackrel{s t}{\geq} \Pi_{\vec{c}_{W}}^{W}$. Apply (28a) to get

$$
Y_{W} \stackrel{s t}{\geq} Y_{W} \wedge X_{W}=Z_{W} \stackrel{s t}{\geq} \Pi_{\vec{c}_{W}}^{W}
$$

and extend this to all of $V$ with the help of lemma 8 .

Proposition 18. Let $\overrightarrow{1} \gg \vec{p} \in \stackrel{\circ}{\mathcal{P}}_{\text {sh }}^{G}$ and $Y \in \mathcal{C}_{G}^{\text {weak }}(\vec{p})$. Let $X$ be $\Pi_{\vec{q}}^{V}$-distributed independently of $Y$ and set $Z:=X \wedge Y$. We claim that for all admissible $(W, v)$

$$
\forall \vec{s}_{W} \in \mathcal{X}_{W}: \quad \mathbb{P}\left(Z_{v}=1 \mid Z_{W}=\vec{s}_{W}\right) \geq q_{v} \alpha_{W}^{v}(\vec{p}) .
$$

Remark. This generalizes [15, proposition 1.2], the core of Liggett, Schonmann \& Stacey's proof, in the following ways: we localize the parameters $\alpha$ and $r$ they used and assume no total ordering of the vertices yet. Furthermore $r_{v}=q_{v}$ follows from a conservative bound of the form

$$
r_{v}:=1-\sup \left\{\alpha_{W}^{v}(\vec{p}):(W, v) \text { escaping }\right\}=1-p_{v}=q_{v},
$$

where the sup is attained in $\alpha_{\emptyset}^{v}(\vec{p})=p_{v}$.

Proof. Recall that $\vec{p} \in \stackrel{\circ}{\mathcal{P}}_{\text {sh }}^{G}$ implies that $\vec{p} \gg \overrightarrow{0}$. Whence $\vec{q} \ll \overrightarrow{1}$ and (35) is well defined because

$$
\forall \text { finite } W \subseteq V, \vec{s}_{W} \in \mathcal{X}_{W}: \quad \mathbb{P}\left(Z_{W}=\vec{s}_{W}\right)>0 .
$$


For every decomposition $N_{0} \uplus N_{1}:=\mathcal{N}(v) \cap W$ with $N_{0}=:\left\{u_{1}, \ldots, u_{l}\right\}$, $N_{1}=:\left\{w_{1}, \ldots, w_{m}\right\}$ and $M:=W \backslash \mathcal{N}(v)$ the fundamental identity (31) implies the inequality

$$
\left[1-\alpha_{W}^{v}(\vec{p})\right]\left(\prod_{j=1}^{l} p_{u_{j}}\right) \prod_{i=1}^{m} \alpha_{M \uplus\left\{w_{1}, \ldots, w_{i-1}\right\}}^{w_{i}}(\vec{p}) \geq q_{v},
$$

where $p_{u_{j}} \geq \alpha_{M \uplus N_{1} \uplus\left\{u_{1}, \ldots, u_{j-1}\right\}}^{u_{j}}(\vec{p})$ follows from (32a).

We prove (35) inductively over the cardinality of $W$. The induction base $W=\emptyset$ is easy as $\mathbb{P}\left(Z_{v}=1\right)=q_{v} \mathbb{P}\left(Y_{v}=1\right) \geq q_{v} p_{v}=q_{v} \alpha_{\emptyset}^{v}(\vec{p})$. For the induction step fix $\vec{s}_{W} \in \mathcal{X}_{W}$ and the decomposition

$$
N_{0}:=\left\{w \in W \cap \mathcal{N}(v): s_{w}=0\right\}=:\left\{u_{1}, \ldots, u_{l}\right\}
$$

and

$$
N_{1}:=\left\{w \in W \cap \mathcal{N}(v): s_{w}=1\right\}=:\left\{w_{1}, \ldots, w_{m}\right\} .
$$

We write

$$
\begin{aligned}
& \mathbb{P}\left(Y_{v}=0 \mid Z_{W}=\vec{s}_{W}\right) \\
= & \mathbb{P}\left(Y_{v}=0 \mid Z_{N_{0}}=\overrightarrow{0}, Z_{N_{1}}=\overrightarrow{1}, Z_{M}=\vec{s}_{M}\right) \\
= & \frac{\mathbb{P}\left(Y_{v}=0, Z_{N_{0}}=\overrightarrow{0}, Z_{N_{1}}=\overrightarrow{1}, Z_{M}=\vec{s}_{M}\right)}{\mathbb{P}\left(Z_{N_{0}}=\overrightarrow{0}, Z_{N_{1}}=\overrightarrow{1}, Z_{M}=\vec{s}_{M}\right)} \\
\leq & \frac{\mathbb{P}\left(Y_{v}=0, Z_{M}=\vec{s}_{M}\right)}{\mathbb{P}\left(X_{N_{0}}=\overrightarrow{0}, Y_{N_{1}}=\overrightarrow{1}, Z_{M}=\vec{s}_{M}\right)} \\
= & \frac{\mathbb{P}\left(Y_{v}=0 \mid Z_{M}=\vec{s}_{M}\right) \mathbb{P}\left(Z_{M}=\vec{s}_{M}\right)}{\mathbb{P}\left(X_{N_{0}}=\overrightarrow{0}\right) \mathbb{P}\left(Y_{N_{1}}=\overrightarrow{1}, Z_{M}=\vec{s}_{M}\right)} \\
\leq & \frac{q_{v}}{\mathbb{P}\left(X_{N_{0}}=\overrightarrow{0}\right) \mathbb{P}\left(Y_{N_{1}}=\overrightarrow{1} \mid Z_{M}=\vec{s}_{M}\right)} \\
= & \frac{q_{v}}{\prod_{j=1}^{l}\left(1-q_{u_{j}}\right) \prod_{i=1}^{m} \mathbb{P}\left(Y_{w_{i}}=1 \mid Y_{w_{1}}=\ldots=Y_{w_{i-1}}=1, Z_{M}=\vec{s}_{M}\right)} \\
\leq & \frac{q_{v}}{\prod_{j=1}^{l} p_{u_{j}} \prod_{i=1}^{m} \alpha_{\left.M \uplus 4 w_{1}, \ldots, w_{i-1}\right\}}^{w_{i}}(\vec{p})} \\
\leq & 1-\alpha_{W}^{v}(\vec{p}) .
\end{aligned}
$$

The key steps in (37) are:

(37a) increasing the numerator by dropping $Z_{N_{0}}=\overrightarrow{0}$ and $Z_{N_{1}}=\overrightarrow{1}$ while decreasing the denominator by using the definition of $Z$,

$(37 \mathrm{c})$ as $d(v, M) \geq 1$ and $Y \in \mathcal{C}_{G}^{\text {weak }}(\vec{p})$,

(37b) using the independence of $X_{N_{0}}$ from $\left(Y_{N_{1}}, Z_{M}\right)$,

(37d) applying the induction hypothesis (35) to the factors of the rhs product in the denominator, which have strictly smaller cardinality,

(37e) applying inequality (36). 
Hence

$$
\mathbb{P}\left(Z_{v}=1 \mid Z_{W}=\vec{s}_{W}\right) \geq q_{v} \mathbb{P}\left(Y_{v}=1 \mid Z_{W}=\vec{s}_{W}\right) \geq q_{v} \alpha_{W}^{v}(\vec{p})
$$

\section{The weak invariant case}

In this section we extend our characterization to the case of BRFs with weak dependency graph, which are invariant under a group action. Let $\Gamma$ be a subgroup of $\operatorname{Aut}(G)$. A BRF $Y$ is $\Gamma$-invariant iff

$$
\forall \gamma \in \Gamma: \quad(\gamma Y):=\left(Y_{\gamma(v)}\right)_{v \in V} \text { has the same law as } Y .
$$

For a given $\Gamma$ and $\Gamma$-invariant $\vec{p}$ we denote by $\mathcal{C}_{\Gamma \text {-inv }}^{\text {weak }}(\vec{p})$ the weak, $\Gamma$-invariant dependency class, that is $\Gamma$-invariant BRFs with weak dependency graph $G$, and by $\mathcal{C}_{\Gamma \text {-inv }}^{\text {strong }}(\vec{p})$ the corresponding strong version.

We call a pair $(G, \Gamma)$ partition exhaustive iff there exists a sequence of partitions $\left(P_{n}\right)_{n \in \mathbb{N}}$ of $V$ with $P_{n}:=\left(V_{i}^{(n)}\right)_{i \in \mathbb{N}}$, such that

$$
\begin{gathered}
\forall n, i, j \in \mathbb{N}: \quad G\left(V_{i}^{(n)}\right) \text { is isomorph to } G\left(V_{1}^{(n)}\right)=: G_{n}, \\
\forall n \in \mathbb{N}: \quad \text { the orbit of } P_{n} \text { under } \Gamma \text { is finite, } \\
V_{1}^{(n)} \underset{n \rightarrow \infty}{\longrightarrow} V, \text { that is }\left(G_{n}\right)_{n \in \mathbb{N}} \text { exhausts } G .
\end{gathered}
$$

The kind of graphs we have in mind are regular infinite trees and tree-like graphs, $\mathbb{Z}^{d}$ and other regular lattices (triangular, hexagonal, ...). We think of the group $\Gamma$ to be generated by some of the natural shifts and rotations of the graph. An example are increasing regular rectangular decompositions of $\mathbb{Z}^{d}$ together with translations of $\mathbb{Z}^{d}$.

Theorem 19. Let $(G, \Gamma)$ be partition exhaustive. Then

$$
\mathcal{P}_{\text {udom }}^{\mathcal{C}_{\Gamma-i n v}^{\text {weak }}}=\mathcal{P}_{\text {dom }}^{\mathcal{C}_{\text {T-inv }}^{\text {weak }}}=\stackrel{\circ}{\mathcal{P}}_{\text {sh }}^{\Gamma-\text { inv }}:=\left\{\vec{p} \in \stackrel{\circ}{\mathcal{P}}_{\text {sh }}^{G}: \quad \vec{p} \text { is } \Gamma \text {-invariant }\right\} .
$$

Remark. It follows from (39) that $\Gamma$ acts quasi-transitively on $G$. Hence $\stackrel{\circ}{\mathcal{P}} \stackrel{\text { sh }}{h}$-inv can be seen as a subset of a finite-dimensional space.

The mixing in (41) destroys strong independence even in simple cases like $G=\mathbb{Z}$ and $\Gamma$ the group of translations of $\mathbb{Z}[15$, end of section 2]. The easiest way to see this is to let $G:=(\{v, w\}, \emptyset), X^{(1)}, X^{(2)} \in \mathcal{C}_{G}^{\text {strong }}(\vec{p})$ and $Y$ be Bernoulli $\left(\frac{1}{2}\right)$-distributed, all independent of each other. Define $Z:=X^{(Y)}$ and ask if $\mathbb{P}\left(Z_{v}=Z_{w}=1\right)=\mathbb{P}\left(Z_{v}=1\right) \mathbb{P}\left(Z_{w}=1\right)$. This fails for most choices of $\vec{p}$. Calculations on slightly more complex graphs as $G=(\{u, v, w\},\{(u, v)\})$ show, that $Z$ from (41) has no strong dependency graph. Thus the present approach, inspired by [15, page 89], does not allow to characterize $\mathcal{P}_{\text {udom }}^{\mathcal{C}_{\text {ring }}^{\text {strong }}}$ and $\mathcal{P}_{\text {dom }}^{\mathcal{C}_{\text {Tinv }}^{\text {strong }}}$.

Proof. As $\mathcal{C}_{\Gamma \text {-inv }}^{\text {weak }}(\vec{p})$ is a subclass of $\mathcal{C}_{G}^{\text {weak }}(\vec{p})$ theorem $(2)$ implies, that $\mathcal{P}_{s h}^{\Gamma \text {-inv }} \subseteq$ $\mathcal{P}_{\text {udom }}^{\mathcal{C}_{\Gamma-\text { inv }}^{\text {weak }}} \subseteq \mathcal{P}_{\text {dom }}^{\mathcal{C}_{\text {Weak }}^{\text {weak }}}$. We show $\mathcal{P}_{d o m}^{\mathcal{C}_{\Gamma-\text { inv }}^{\text {weak }}} \subseteq \mathcal{P}_{\text {sh }}^{\Gamma \text {-inv }}$ by constructing a counterexample. 
If $\vec{p} \notin \stackrel{\circ}{\mathcal{P}_{s h}^{\Gamma-i n v}}$, then by (39c) there exists a $n \in \mathbb{N}$, such that $\vec{p} \notin \stackrel{\circ}{\mathcal{P}}_{s h}^{G_{n}, \Gamma}$ (the intersection of the projections of $\Gamma$-invariant parameters on $G$ with $\left.\mathcal{P}_{s h}^{\circ} G_{n}\right)$. Let $P:=P_{n}$ and let $\left(P^{(1)}, \ldots, P^{(k)}\right)$ be its finite orbit under the action of $\Gamma(39 \mathrm{~b})$. By (39a) each class $V_{(i, j)} \in P^{(j)}$ has a graph $G\left(V_{(i, j)}\right)$ isomorph to $G_{n}$. Use lemma 11 to construct i.i.d. BPFs $Z^{(i, j)} \in \mathcal{C}_{G_{n}}^{\text {strong }}(\vec{p})$ with $\mathbb{P}\left(Z^{(i, j)}=\overrightarrow{1}\right)=0$. For $j \in[k]$, collate the $Z_{(i, j)}$ to a $\mathrm{BPF} Z^{(j)}$. This works, as $P^{(j)}$ is a partition of $G$. By definition $Z^{(j)} \in \mathcal{C}_{\Gamma \text {-inv }}^{\text {strong }}(\vec{p})$. Finally let $U$ be Uniform $([k])$-distributed and independent of everything else. Define the final BPF $Z$ by

$$
Z:=\sum_{j=1}^{k}[U=j] Z^{(j)}
$$

We claim that $Z \in \mathcal{C}_{\Gamma \text {-inv }}^{\text {weak }}(\vec{p})$. The mixing in $(41)$ keeps $Z \in \mathcal{C}_{G}^{\text {weak }}(\vec{p})$. To see its $\Gamma$-invariance, let $\gamma \in \Gamma$. The automorphism $\gamma$ acts injectively on $\left(P^{(1)}, \ldots, P^{(k)}\right)$ and thus also on $[k]$. Therefore, using the fact that $U$ is uniform and everything is constructed independently, we have

$$
\gamma Z=\sum_{j=1}^{k}[U=j] \gamma Z^{(j)}=\sum_{j=1}^{k}\left[U=\gamma^{-1} j\right] Z^{(j)}=\sum_{j=1}^{k}[U=j] Z^{(j)}=Z .
$$

\section{The asymptotic size of the jump on $\mathbb{Z}_{(k)}$}

Liggett, Schonmann \& Stacey formulated the following conjecture about the size of the jump at the critical value on $\mathbb{Z}_{(k)}$, the $k$-fuzz of $\mathbb{Z}$ :

Conjecture 20 ([15, after corollary 2.2]).

$$
\forall k \in \mathbb{N}_{0}: \quad \sigma\left(\mathcal{C}_{\mathbb{Z}_{(k)}^{\text {weak }}}\left(p_{\text {udom }}^{\mathcal{C}_{(k)}^{\text {weak }}}\right)\right)=\frac{k}{k+1} .
$$

We think that Liggett, Schonmann \& Stacey were led by the intuition, that the extra randomness used in obtaining the above lower bound (see the $Y$ in [15, proposition 1.2] or the $X$ in the proof of proposition 18) can be ignored in a suitable transitive setting. This would, in general, yield $\sigma\left(\mu_{G, p_{s h}^{G}}\right)=$ $\sigma\left(\mathcal{C}_{G}^{\text {weak }}\left(p_{\text {udom }}^{\mathcal{C}_{G}^{\text {weak }}}\right)\right)$, and, in the particular case of $\mathbb{Z}_{(k)}, \sigma\left(\mu_{\mathbb{Z}_{(k)}, p_{s h}^{\mathbb{Z}_{(k)}}}\right)=\frac{k}{k+1}[16$, section 4.2], with $p_{s h}^{\mathbb{Z}_{(k)}}=1-\frac{k^{k}}{(k+1)^{(k+1)}}$ [16, section 4.2$]$.

Proposition 21 shows, that asymptotically $\sigma\left(\mathcal{C}_{\mathbb{Z}_{(k)}^{\text {weak }}}\left(p_{s h}^{\mathbb{Z}_{(k)}}\right)\right)$ is much closer to the lower bound of $\frac{k}{(k+1)^{2}}$ from [15, corollary 2.5]. This is caused by the increasing range of dependence, as $k \rightarrow \infty$, which allows for extreme correlations on the same order as the extra randomness used to decorrelate them.

Proposition 21. For $k \in \mathbb{N}$ and $\Gamma_{k}$ the translations of $\mathbb{Z}$, let $C_{k}$ be either $\mathcal{C}_{\mathbb{Z}_{(k)}}^{\text {strong }}$ or $\mathcal{C}_{\Gamma_{k} \text {-inv }}^{\text {weak }}$. We have

$$
\forall \varepsilon>0: \exists K(\varepsilon): \forall k \geq K: \quad \sigma\left(C_{k}\left(p_{s h}^{\mathbb{Z}_{(k)}}\right)\right) \leq \frac{1+(1+\varepsilon) \ln (k+1)}{k+1} .
$$


Proof. Let $\mathcal{N}_{1}(0)^{+}:=\{0, \ldots, k\}$ be the non-negative closed half-ball of radius $k$ centred at 0 . Define a BRF $Y$ on $\mathbb{Z}$ by setting $\mathbb{P}\left(Y_{\mathcal{N}_{1}(0)^{+}}=\overrightarrow{1}\right):=p_{\text {dom }}^{\mathbb{Z}_{(k)}}$, $\mathbb{P}\left(Y_{\mathcal{N}_{1}(0)^{+}}=\overrightarrow{0}\right):=q_{d o m}^{\mathbb{Z}_{(k)}}$ and letting $Y_{\mathbb{Z} \backslash \mathcal{N}_{1}(0)^{+}}$be $\prod_{p_{d o m}}^{\prod_{\mathbb{Z}_{(k)}}^{\mathbb{Z} \backslash \mathcal{N}_{1}(0)^{+}}}$-distributed independently of $Y_{\mathcal{N}_{1}(0)^{+}}$. As $Y \in \mathcal{C}_{\mathbb{Z}_{(k)}}^{\text {strong }}\left(p_{d o m}^{\mathbb{Z}_{(k)}}\right)$, [15, corollary 2.5] applies and $Y \stackrel{s t}{\geq} X$, where $X$ is $\Pi_{\sigma}^{\mathbb{Z}}$-distributed with $\sigma \in\left[\frac{k}{(k+1)^{2}}, \frac{k}{k+1}\right]$. Lemma 8 implies $X_{\mathcal{N}_{1}(\overrightarrow{0})}+\stackrel{s t}{\leq} Y_{\mathcal{N}_{1}(\overrightarrow{0})}+$ and in particular the inequality

$$
1-(1-\sigma)^{(k+1)}=\mathbb{P}\left(X_{\mathcal{N}_{1}(\overrightarrow{0})^{+}} \neq \overrightarrow{0}\right)=\mathbb{P}\left(Y_{\mathcal{N}_{1}(\overrightarrow{0})^{+}} \neq \overrightarrow{0}\right)=1-\frac{k^{k}}{(k+1)^{(k+1)}} .
$$

Rewrite it into

$$
\begin{aligned}
\sigma & \leq 1-\frac{k^{\frac{k}{k+1}}}{k+1} \\
& =\frac{1}{k+1}+\frac{k}{k+1}\left(1-k^{-\frac{1}{k+1}}\right) \\
& \leq \frac{1}{k+1}+\left(1-(k+1)^{-\frac{1}{k+1}}\right) .
\end{aligned}
$$

For every $\varepsilon>0$ and $z$ close enough to 0 we know that $1-e^{-z} \leq(1+\varepsilon) z$. The statement for $\mathcal{C}_{\mathbb{Z}_{(k)}}^{\text {strong }}$ follows from $z_{k}:=\frac{\ln (k+1)}{k+1} \underset{k \rightarrow \infty}{\longrightarrow} 0$. The result for $\mathcal{C}_{\Gamma_{k} \text {-inv }}^{\text {weak }}$ follows from a mixing construction similar to (41).

\section{Acknowledgements}

I want to thank Yuval Peres and Rick Durrett for pointing out [15] to me and Pierre Mathieu for listening patiently to my numerous attempts at understanding and solving this problem. This work has been partly done during a series of stays at the LATP, Aix-Marseille Université, financially supported by grants A3-16.M-93/2009-1 and A3-16.M-93/2009-2 from the Land Steiermark and by the Austrian Science Fund (FWF), project W1230-N13. I am also indebted to the anonymous referees for their constructive comments.

\section{References}

[1] E. D. Andjel. Characteristic exponents for two-dimensional bootstrap percolation. Ann. Probab., 21(2):926-935, 1993.

[2] P. Antal and A. Pisztora. On the chemical distance for supercritical Bernoulli percolation. Ann. Probab., 24(2):1036-1048, 1996.

[3] P. Billingsley. Probability and measure. Wiley Series in Probability and Mathematical Statistics: Probability and Mathematical Statistics. John Wiley \& Sons Inc., New York, third edition, 1995.

[4] R. Bissacot, R. Fernández, and A. Procacci. On the convergence of cluster expansions for polymer gases. J. Stat. Phys., 139(4):598-617, 2010. 
[5] R. Bissacot, R. Fernández, A. Procacci, and B. Scoppola. An improvement of the Lovász Local Lemma via cluster expansion. Combinatorics, Probability and Computing, 2011. Available on CJO.

[6] B. Bollobás and O. Riordan. Percolation. Cambrigde University Press, 2006.

[7] R. L. Dobrushin. Perturbation methods of the theory of Gibbsian fields. In Lectures on probability theory and statistics (Saint-Flour, 1994), volume 1648 of Lecture Notes in Math., pages 1-66. Springer, Berlin, 1996.

[8] P. Erdős and L. Lovász. Problems and results on 3-chromatic hypergraphs and some related questions. In Infinite and finite sets (Colloq., Keszthely, 1973; dedicated to P. Erdös on his 60th birthday), Vol. II, pages 609-627. Colloquia Mathematica Societatis János Bolyai, Vol. 10. North-Holland, Amsterdam, 1975.

[9] R. Fernández and A. Procacci. Cluster expansion for abstract polymer models. New bounds from an old approach. Comm. Math. Phys., 274(1):123140, 2007.

[10] D. C. Fisher and A. E. Solow. Dependence polynomials. Discrete Math., 82(3):251-258, 1990.

[11] G. Grimmett. Percolation, volume 321 of Grundlehren der Mathematischen Wissenschaften [Fundamental Principles of Mathematical Sciences]. Springer-Verlag, Berlin, second edition, 1999.

[12] C. Gruber and H. Kunz. General properties of polymer systems. Comm. Math. Phys., 22:133-161, 1971.

[13] C. Hoede and X. L. Li. Clique polynomials and independent set polynomials of graphs. Discrete Math., 125(1-3):219-228, 1994. 13th British Combinatorial Conference (Guildford, 1991).

[14] T. M. Liggett. Interacting particle systems. Classics in Mathematics. Springer-Verlag, Berlin, 2005. Reprint of the 1985 original.

[15] T. M. Liggett, R. H. Schonmann, and A. M. Stacey. Domination by product measures. Ann. Probab., 25(1):71-95, 1997.

[16] P. Mathieu and C. Temmel. K-independent percolation on trees. Stochastic processes and applications, 2012.

[17] L. Russo. An approximate zero-one law. Z. Wahrsch. Verw. Gebiete, 61(1):129-139, 1982.

[18] A. D. Scott and A. D. Sokal. The repulsive lattice gas, the independent-set polynomial, and the Lovász local lemma. J. Stat. Phys., 118(5-6):1151$1261,2005$.

[19] J. B. Shearer. On a problem of Spencer. Combinatorica, 5(3):241-245, 1985. 
[20] V. Strassen. The existence of probability measures with given marginals. Ann. Math. Statist., 36:423-439, 1965.

[21] C. Temmel. Shearer's measure and stochastic domination of product measures. submitted, 2011.

\section{Additional Material}

\subsection{Intrinsic coupling and domination of Shearer's mea- sure}

In this section take a look at the parameters of the BPF dominated by Shearer's measure. We specialize proposition 18 in proposition 22 and find that we do not need an auxiliary BPF. Therefore a natural vector in the set $\Sigma\left(\mu_{G, \vec{p}}\right)$ is described by the the one-vertex open extensions probabilities in proposition 23. We only deal with connected graphs, as the results factorize over connected components.

The vertex-wise max operation is defined analogously to the vertex-wise minimum (27). It erases 0s in realizations, thinning out independent sets of $0 \mathrm{~s}$. Hence it conserves Shearer's measure. Formally, let $Y$ be $\mu_{G, \vec{p}}$-distribued and

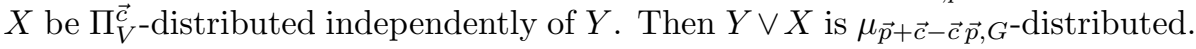
This is a coupling between $\mu_{\vec{p}, V}$ and $\mu_{\vec{p}+\vec{c}-\vec{c} \vec{p}, G}$ and implies that

$$
\mu_{\vec{p}, V} \stackrel{s t}{\leq} \mu_{\vec{p}+\vec{c}-\vec{c} \vec{p}, G},
$$

with equality iff $X=0$. Furthermore

$$
\forall(W, v): \quad \alpha_{W}^{v}(\vec{p}+\vec{c}-\vec{c} \vec{p})=\alpha_{W}^{v}(\vec{p})+c_{v}\left[1-\alpha_{W}^{v}(\vec{p})\right] \geq \alpha_{W}^{v}(\vec{p}) .
$$

This implies the monotonicity of $\Xi_{G}$ on $\mathcal{P}_{s h}^{G}$, the fact that $\mathcal{P}_{s h}^{G}$ and $\stackrel{\circ}{\mathcal{P}}_{\text {sh }}^{G}$ are up-sets and the monotonicity of $\vec{x}$ from (46a) in $\vec{p}$ with $\lim _{\vec{p} \rightarrow \overrightarrow{1}} \vec{x}=\overrightarrow{1}$.

Proposition 22. Let $G:=(V, E)$ be connected, $\vec{p} \in \stackrel{\circ}{\mathcal{P}}_{s h}^{G}$ and $Y$ be $\mu_{G, \vec{p}^{-}}$ distributed. We claim that for all admissible pairs $(W, v)$

$$
\forall \vec{s}_{W} \in \mathcal{X}_{W}: \quad \mu_{G, \vec{p}}\left(Y_{v}=1 \mid Y_{W}=\vec{s}_{W}\right) \geq \alpha_{W}^{v}(\vec{p})>0 .
$$

Proof. The fact that $\vec{p} \in \stackrel{\circ}{\mathcal{P}}_{s h}^{G}$ implies that all admissible $\alpha_{W}^{v}(\vec{p})$ are well defined and non-zero.

We prove (45) inductively over the cardinality of $W$. The induction base for $W=\emptyset$ is $\mu_{G, \vec{p}}\left(Y_{v}=1\right)=p_{v}=\alpha_{\emptyset}^{v}(\vec{p})$. For the induction step let $M:=W \backslash \mathcal{N}(v)$ and $N:=W \cap \mathcal{N}(v)$. Let $\vec{s}_{W} \in \mathcal{X}_{W}$ and assume that $\mu_{G, \vec{p}}\left(Y_{W}=\vec{s}_{W}\right)>0$. The first case is $\vec{s}_{N} \neq \overrightarrow{1}$, whereby

$$
\mu_{G, \vec{p}}\left(Y_{v}=0 \mid Y_{W}=\vec{s}_{W}\right)=\frac{\mu_{G, \vec{p}}\left(Y_{v}=0, Y_{N} \neq \overrightarrow{1}, Y_{M}=\vec{s}_{M}\right)}{\mu_{G, \vec{p}}\left(Y_{Y}=\vec{s}_{W}\right)}=0,
$$

as there are neighbouring zeros in $\left(Y_{v}, Y_{N}\right)$. The second case is $\vec{s}_{N}=\overrightarrow{1}$. Let $\left\{w_{1}, \ldots, w_{m}\right\}:=N$. Use the fundamental identity (31) to get

$$
\mu_{G, \vec{p}}\left(Y_{v}=0 \mid Y_{W}=\vec{s}_{W}\right)
$$




$$
\begin{aligned}
& =\frac{\mu_{G, \vec{p}}\left(Y_{v}=0, Y_{N}=\overrightarrow{1}, Y_{M}=\vec{s}_{M}\right)}{\mu_{G, \vec{p}}\left(Y_{N}=\overrightarrow{1}, Y_{M}=\vec{s}_{M}\right)} \\
& =\frac{\mu_{G, \vec{p}}\left(Y_{v}=0\right) \mu_{G, \vec{p}}\left(Y_{M}=\vec{s}_{M}\right)}{\mu_{G, \vec{p}}\left(Y_{N}=\overrightarrow{1}, Y_{M}=\vec{s}_{M}\right)} \\
& =\frac{q_{v}}{\mu_{G, \vec{p}}\left(Y_{N}=\overrightarrow{1} \mid Y_{M}=\vec{s}_{M}\right)} \\
& =\frac{q_{v}}{\prod_{i=1}^{m} \mu_{G, \vec{p}}\left(Y_{w_{i}}=1 \mid Y_{\left\{w_{1}, \ldots, w_{i-1}\right\}}=\overrightarrow{1}, Y_{M}=\vec{s}_{M}\right)} \\
& \leq \frac{q_{v}}{\prod_{i=1}^{m} \alpha_{M \uplus\left\{w_{1}, \ldots, w_{i-1}\right\}}^{w_{i}}(\vec{p})} \\
& =1-\alpha_{W}^{v}(\vec{p}) .
\end{aligned}
$$

Proposition 23. Let $G$ be infinite and connected. Assume that $\vec{p} \in \stackrel{\circ}{\mathcal{P}}_{\text {sh }}^{G}$. Define the vector $\vec{x}$ by

$$
\forall v \in V: \quad x_{v}:=\inf \left\{\alpha_{W}^{v}(\vec{p}):(W, v) \text { is escaping }\right\} .
$$

Then

$$
\mu_{G, \vec{p}} \geq \Pi_{\vec{x}}^{V}, \quad \text { that is } \vec{x} \in \Sigma\left(\mu_{G, \vec{p}}\right)
$$

and

$$
\forall v \in V: \quad x_{v} \geq \min \left\{q_{w}: w \in \mathcal{N}(v)\right\}>0 .
$$

Proof. This is the same proof as the one for proposition 17, except that instead of using the auxiliary BPF $X$ and (35) from proposition 18 we use (45) from proposition 22 directly.

Proposition 24. Let $G:=\mathbb{Z}^{2}$. Define the sets

$$
W_{(n, k, l)}:=\{(x, y): 0 \leq x<n, 0 \leq y<k+l\} \uplus\{x=n, 0 \leq y<k\}
$$

and the value

$$
a(p):=\inf \left\{\alpha_{W_{(n, k, l)}^{(n, k)}}^{(p)}\right\} \geq q .
$$

Then, with $G_{N}$ being the subgraph induced by $V_{N}:=\{(x, y): 0 \leq x, y<N\}$, we have

This implies that

$$
\lim _{N \rightarrow \infty} \frac{\log \Xi_{G_{N}}(p)}{N^{2}}=\log a(p)
$$

$$
\sigma\left(\mu_{\mathbb{Z}^{2}, p}\right)=a(p) \geq q_{s h}^{\mathbb{Z}^{2}}>0 .
$$

Remark. The result of proposition 24 should be easily generalizable to $\mathbb{Z}^{d}$ and other $d$-dimensional transitive lattice like graphs. I even go so far as to conjecture that something similar should hold on all infinite, locally finite quasitransitive graphs with quasi-transitive parameters. The obstacle seems mostly notational, especially in writing down a nice subset of escaping $(W, v)$ s exhaust$\operatorname{ing} V$.

Contrast the proof of proposition 24 with the subadditive approach in $[18$, section 8.3]. 
Proof. We see that for $(n, k, l) \leq(\bar{n}, \bar{k}, \bar{l})$ we have

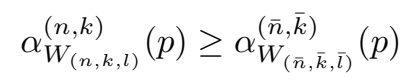

and hence

$$
a(p)=\lim _{n, k . l \rightarrow \infty} \alpha_{W_{(n, k, l)}}^{(n, k)}(p)
$$

is well-defined.

Enumerate $V_{N}=:\left\{v_{1}, \ldots, v_{N^{2}}\right\}$ starting from $\left(\left\lfloor\frac{N}{2}\right\rfloor,\left\lfloor\frac{N}{2}\right\rfloor\right)$ stepping to the left for the first step and spiraling outwards anti-clockwise around $\left(\left\lfloor\frac{N}{2}\right\rfloor,\left\lfloor\frac{N}{2}\right\rfloor\right)$. Setting $W_{i}:=\left\{v_{1}, \ldots, v_{i-1}\right\}$ we have

$$
\Xi_{G_{N}}(p)=\prod_{i=1}^{N^{2}} \alpha_{W_{i}}^{v_{i}}(p)=\prod_{i=1}^{N^{2}} \alpha_{W_{\left(n_{i}, k_{i}, l_{i}\right)}^{\left(n_{i}, k_{i}\right)}}^{(p)} .
$$

Thus on the one hand we have

$$
\Xi_{G_{N}}(p) \geq a(p)^{N^{2}}
$$

On the other hand choose $\varepsilon>0$. Then there exists a $(n, k . l)$ such that $\alpha_{W_{(n, k, l)}}^{(n, k)}(p) \leq a(p)+\varepsilon$. We estimate roughly

$$
\begin{aligned}
& \Xi_{G_{N}}(p)=\Xi_{G\left(W_{(n, k, l)}\right)}(p) \prod_{i=(n \vee(k+l+1))^{2}}^{N^{2}} \alpha_{W_{\left(n_{i}, k_{i}, l_{i}\right)}}^{\left(n_{i}, k_{i}\right)}(p) \\
& \leq \Xi_{G\left(W_{(n, k, l)}\right)}(p)(a(p)+\varepsilon)^{N^{2}-(n \vee(k+l+1))^{2}-4 N(k+l)}
\end{aligned}
$$

Therefore

$$
a(p)^{N^{2}} \leq \Xi_{G_{N}}(p) \leq \Xi_{G\left(W_{(n, k, l)}\right)}(p)(a(p)+\varepsilon)^{N^{2}-(n \vee(k+l+1))^{2}-4 N(k+l)}
$$

and

$$
\begin{aligned}
& \log a(p) \leq \frac{\log \Xi_{G_{N}}(p)}{N^{2}} \\
& \leq \frac{\log \Xi_{G\left(W_{(n, k, l)}\right)}(p)}{N^{2}}+\frac{N^{2}-(n \vee(k+l+1))^{2}-4 N(k+l)}{N^{2}} \log (a(p)+\varepsilon),
\end{aligned}
$$

resulting in $(47 \mathrm{c})$ by taking the limit.

For (47d) adapt the reasoning from proposition 23 to the escaping $\alpha_{W_{(n, k, l)}}^{(n, k)}(p)$, do the coupling from (44) and the estimate from theorem 4 to get a lower bound $\sigma\left(\mu_{\mathbb{Z}^{2}, p}\right) \geq \sigma\left(\mu_{\mathbb{Z}^{2}, p_{s h}^{Z^{2}}}\right) \geq q_{s h}^{\mathbb{Z}^{2}}>0$. The upper bound follows straight from (47c) (use the monotone functions $f_{W}:=\mathbb{I}_{\overrightarrow{1}_{W}}$ ). 


\subsection{Tools for stochastic domination II}

This section contains a number of proofs omitted in section 5.1 as well as some additional comments regarding stochastic domination of BRFs.

For $W \subset V$ and $\vec{s}_{W} \in \mathcal{X}_{W}$ we define the cylinder set $\Pi_{W}^{-1}\left(\vec{s}_{W}\right)$ by

$$
\Pi_{W}^{-1}\left(\vec{s}_{W}\right):=\left\{\vec{t} \in \mathcal{X}_{V}: \vec{t}_{W}=\vec{s}_{W}\right\} .
$$

Lemma 25 ([14, chapter II, theorem 2.4]). Let $Y, Z$ be two BRFs indexed by $V$, then $Y \geq Z$ iff there exists a $\nu \in \mathcal{M}_{1}\left(\mathcal{X}_{V}^{2}\right)$ such that

$$
\begin{gathered}
\forall \text { finite } W \subseteq V, \forall \vec{s}_{W} \in \mathcal{X}_{W}: \quad \nu\left(\Pi_{W}^{-1}\left(\vec{s}_{W}\right) \times \mathcal{X}_{V}\right)=\mathbb{P}\left(Y_{W}=\vec{s}_{W}\right) \\
\forall \text { finite } W \subseteq V, \forall \vec{t}_{W} \in \mathcal{X}_{W}: \quad \nu\left(\mathcal{X}_{V} \times \Pi_{W}^{-1}\left(\vec{t}_{W}\right)\right)=\mathbb{P}\left(Z_{W}=\vec{t}_{W}\right) \\
\nu\left(\left\{(\vec{s}, \vec{t}) \in \mathcal{X}_{V}^{2}: \vec{s} \geq \vec{t}\right\}\right)=1 .
\end{gathered}
$$

Remark. The coupling probability measure $\nu$ in lemma 25 is in general not unique.

Proposition 26. Let $Y$ and $Z$ be two BRFs indexed by the same set $V$. Then we have:

$$
Y \stackrel{\text { st }}{\geq} \Rightarrow \forall \text { finite } W \subseteq V:\left(\begin{array}{c}
\mathbb{P}\left(Y_{W}=\overrightarrow{1}\right) \geq \mathbb{P}\left(Z_{W}=\overrightarrow{1}\right) \\
\text { and } \\
\mathbb{P}\left(Y_{W}=\overrightarrow{0}\right) \leq \mathbb{P}\left(Z_{W}=\overrightarrow{0}\right)
\end{array}\right)
$$

Proof. Assume that $Y \stackrel{s t}{\geq} Z$ and let $W \subseteq V$ be finite. Lemma 8 asserts that $Y_{W} \stackrel{s t}{\geq} Z_{W}$. Regard the monotone functions $f=\mathbb{I}_{\Pi_{W}^{-1}(\overrightarrow{1})}$ and $g=1-\mathbb{I}_{\Pi_{W}^{-1}(\overrightarrow{0})}$. Stochastic domination implies that

$$
\mathbb{P}\left(Y_{W}=\overrightarrow{1}\right)=\mathbb{E}[f(Y)] \geq \mathbb{E}[f(Z)]=\mathbb{P}\left(Z_{W}=\overrightarrow{1}\right)
$$

and

$$
\mathbb{P}\left(Y_{W}=\overrightarrow{0}\right)=1-\mathbb{E}[g(Y)] \leq 1-\mathbb{E}[g(Z)]=\mathbb{P}\left(Z_{W}=\overrightarrow{0}\right)
$$

Proposition 27. Let $Y$ be a BRF taking values in $\mathcal{X}_{V}$. Then $\Sigma(Y)$ is closed and a down-set.

Proof. Take a finite $W \subseteq V$. Then $\Sigma\left(Y_{W}\right)$ is closed because we have a finite number of inequalities over the space of probability measures on $\mathcal{X}_{W}$, which is at most $2^{|W|}$-dimensional. If $\vec{c} \in \Sigma\left(Y_{W}\right)$ and $\vec{d} \leq \vec{c}$, then $\Pi_{\vec{d}}^{W} \stackrel{s t}{\leq} \Pi_{\vec{c}}^{W} \stackrel{s t}{\leq} Y$. Therefore $\Sigma\left(Y_{W}\right)$ is a down-set. Those properties then carry over to $\Sigma(Y)$ by taking the limit in the net of finite subsets of $V$.

Proof. (of (28)) Take a finite $W \subseteq V$ and $f \in \operatorname{Mon}(W)$. Then

$$
\begin{aligned}
& \mathbb{E}\left[f\left(Y_{W} \wedge Z_{W}\right)\right] \\
= & \sum_{\vec{z} \in \operatorname{supp} Z_{W}} \mathbb{E}\left[f\left(Y_{W} \wedge \vec{z}\right) \mid Z_{W}=\vec{z}\right] \mathbb{P}\left(Z_{W}=\vec{z}\right)
\end{aligned}
$$




$$
\begin{aligned}
& \leq \sum_{\vec{z} \in \operatorname{supp} Z_{W}} \mathbb{E}\left[f\left(Y_{W}\right) \mid Z_{W}=\vec{z}\right] \mathbb{P}\left(Z_{W}=\vec{z}\right) \\
& =\mathbb{E}\left[f\left(Y_{W}\right)\right] \\
& =\sum_{\vec{z} \in \operatorname{supp} Z_{W}} \mathbb{E}\left[f\left(Y_{W}\right) \mid Z_{W}=\vec{z}\right] \mathbb{P}\left(Z_{W}=\vec{z}\right) \\
& \leq \sum_{\vec{z} \in \operatorname{supp} Z_{W}} \mathbb{E}\left[f\left(Y_{W} \vee \vec{z}\right) \mid Z_{W}=\vec{z}\right] \mathbb{P}\left(Z_{W}=\vec{z}\right) \\
& =\mathbb{E}\left[f\left(Y_{W} \vee Z_{W}\right)\right] .
\end{aligned}
$$

Hence $Y_{W} \wedge Z_{W} \stackrel{s t}{\leq} Y_{W} \stackrel{s t}{\leq} Y_{W} \vee Z_{W}$. For $\vec{x} \in \mathcal{X}_{W}$ and $f \in \operatorname{Mon}(W)$ define

$$
f_{\vec{x}}: \quad \mathcal{X}_{W} \rightarrow \mathbb{R} \quad \vec{y} \mapsto f(\vec{y} \wedge \vec{x}) .
$$

Then $f_{\vec{x}} \in \operatorname{Mon}(W)$, as

$$
\vec{y} \leq \vec{z} \Rightarrow \vec{y} \vee \vec{x} \leq \vec{z} \vee \vec{x} \Rightarrow f_{\vec{x}}(\vec{y})=f(\vec{y} \vee \vec{x}) \leq f(\vec{z} \vee \vec{x})=f_{\vec{z}}(\vec{y}) .
$$

We get

$$
\begin{aligned}
& \mathbb{E}\left[f\left(Y_{W} \vee X_{W}\right)\right] \\
= & \sum_{\vec{x} \in \mathcal{X}_{W}} \mathbb{E}\left[f\left(Y_{W} \wedge \vec{x}\right)\right] \mathbb{P}\left(X_{W}=\vec{x}\right) \\
= & \sum_{\vec{x} \in \mathcal{X}_{W}} \mathbb{E}\left[f_{\vec{x}}\left(Y_{W}\right)\right] \mathbb{P}\left(X_{W}=\vec{x}\right) \\
\geq & \sum_{\vec{x} \in \mathcal{X}_{W}} \mathbb{E}\left[f_{\vec{x}}\left(Z_{W}\right)\right] \mathbb{P}\left(X_{W}=\vec{x}\right) \quad \text { as } Y_{W} \geq Z_{W} \text { and } f \in \operatorname{Mon}(W) \\
= & \mathbb{E}\left[f\left(Z_{W} \vee X_{W}\right)\right] .
\end{aligned}
$$

The same derivation holds for $\wedge$ instead of $\vee$. Note that the fact that $X$ is independent of $(Y, Z)$ is crucial, as we do not know if $Y_{W}\left|X=\vec{x} \geq \stackrel{s t}{\geq} Z_{W}\right| X=\vec{x}$. Finally (28) results from applying lemma 8.

Proof. (of proposition 9) We show that $\nu$ fulfills the conditions of (25). During this proof we interpret $[0]$ as $\emptyset$. We define a probability measure $\nu$ on $\mathcal{X}_{\mathbb{N}^{2}}$ inductively by:

$$
\begin{aligned}
& \forall n \geq 1, \forall \vec{s}_{[n-1]}, \vec{t}_{[n-1]} \in \mathcal{X}_{[n-1]}, \forall a, b \in\{0,1\}: \\
& \nu\left(\Pi_{\{n\}}^{-1}(a) \times \Pi_{\{n\}}^{-1}(b) \mid \Pi_{[n-1]}^{-1}\left(\vec{s}_{[n-1]}\right) \times \Pi_{[n-1]}^{-1}\left(\vec{t}_{[n-1]}\right)\right) \\
& := \begin{cases}=\mathbb{P}\left(Z_{n}=1 \mid Z_{[n-1]}=\vec{s}_{[n-1]}\right) & \text { if }(a, b)=(1,1) \\
=0 & \text { if }(a, b)=(1,0) \\
=p_{n}-\mathbb{P}\left(Z_{n}=1 \mid Z_{[n-1]}=\vec{s}_{[n-1]}\right) & \text { if }(a, b)=(0,1) \\
=1-p_{n} & \text { if }(a, b)=(0,0) .\end{cases}
\end{aligned}
$$

A straightforward induction over $n$ shows that $\nu$ is a probability measure. The induction base is

$\sum_{s_{1}, t_{1}} \nu\left(\Pi_{\{1\}}^{-1}\left(s_{1}\right) \times \Pi_{\{1\}}^{-1}\left(t_{1}\right)\right)=\left(1-p_{1}\right)+\left(p_{1}-\mathbb{P}\left(Z_{1}=1\right)\right)+0+\mathbb{P}\left(Z_{1}=1\right)=1$. 
The induction step is

$$
\begin{aligned}
& \sum_{\vec{s}_{[n]}, \vec{t}_{[n]}} \nu\left(\Pi_{[n]}^{-1}\left(\vec{s}_{[n]}\right) \times \Pi_{[n]}^{-1}\left(\vec{t}_{[n]}\right)\right) \\
= & \sum_{\vec{s}_{[n-1]}, \vec{t}_{[n-1]}} \nu\left(\Pi_{[n]}^{-1}\left(\vec{s}_{[n]}\right) \times \Pi_{[n]}^{-1}\left(\vec{t}_{[n]}\right)\right) \\
\times & \underbrace{\left(\sum_{s_{n}, t_{n}} \nu\left(\Pi_{\{n\}}^{-1}\left(s_{n}\right) \times \Pi_{\{n\}}^{-1}\left(t_{n}\right) \mid \Pi_{[n-1]}^{-1}\left(\vec{s}_{[n-1]}\right) \times \Pi_{[n-1]}^{-1}\left(\vec{t}_{[n-1]}\right)\right)\right)}_{=1 \text { by definition of } \nu} \\
= & \underbrace{\sum_{\vec{s}_{[n-1]}, \vec{t}_{[n-1]}} \nu\left(\Pi_{[n]}^{-1}\left(\vec{s}_{[n]}\right) \times \Pi_{[n]}^{-1}\left(\vec{t}_{[n]}\right)\right)}_{=1 \text { by induction }} .
\end{aligned}
$$

Next we calculate its marginals. Let $n \geq 1$ and $\vec{s}_{[n]} \in \mathcal{X}_{[n]}$. Then we have

$$
\begin{aligned}
& \nu\left(\Pi_{[n]}^{-1}\left(\vec{s}_{[n]}\right) \times \mathcal{X}_{\mathbb{N}}\right) \\
= & \prod_{i=1}^{n} \nu\left(\Pi_{\{i\}}^{-1}\left(\vec{s}_{i}\right) \times \mathcal{X}_{\mathbb{N}} \mid \Pi_{[i-1]}^{-1}\left(\vec{s}_{[i-1]}\right) \times \mathcal{X}_{\mathbb{N}}\right) \\
= & \prod_{i=1}^{n} \mathbb{P}\left(Z_{i}=s_{i} \mid Z_{[i-1]}=\vec{s}_{[i-1]}\right) \\
= & \mathbb{P}\left(Z_{[n]}=\vec{s}_{[n]}\right)
\end{aligned}
$$

and

$$
\begin{aligned}
& \nu\left(\mathcal{X}_{\mathbb{N}} \times \Pi_{[n]}^{-1}\left(\vec{s}_{[n]}\right)\right) \\
= & \prod_{i=1}^{n} \nu\left(\mathcal{X}_{\mathbb{N}} \times \Pi_{\{i\}}^{-1}\left(s_{i}\right) \mid \mathcal{X}_{\mathbb{N}} \times \Pi_{[i-1]}^{-1}\left(\vec{s}_{[i-1]}\right)\right) \\
= & \prod_{i=1}^{n}\left[\left(1-p_{i}\right) \mathbb{I}_{\{0\}}\left(s_{i}\right)+p_{i} \mathbb{I}_{\{1\}}\left(s_{i}\right)\right] \\
= & \mathbb{P}\left(X_{[n]}=\vec{s}_{[n]}\right) .
\end{aligned}
$$

Hence the marginal of the first coordinate has the same law as $Z$ and the marginal of the second coordinate has the law $\Pi_{\vec{p}}^{\mathbb{N}}$.

Finally we calculate $(49 \mathrm{c})$ for $\nu$. We proceed by induction over $n$. The induction base is

$$
\nu\left(\left\{(\vec{s}, \vec{t}) \in \mathcal{X}_{V}^{2}: \vec{s}_{1} \geq \vec{t}_{1}\right\}\right)=\nu\left(\left\{(\vec{s}, \vec{t}) \in \mathcal{X}_{V}^{2}: \vec{s}_{1}=0<\vec{t}_{1}=1\right\}\right)=0 .
$$

The induction step is

$$
\begin{aligned}
& \nu\left(\left\{(\vec{s}, \vec{t}) \in \mathcal{X}_{V}^{2}: \vec{s}_{[n]} \geq \vec{t}_{[n]}\right\}\right) \\
= & \underbrace{\nu\left(\left\{(\vec{s}, \vec{t}) \in \mathcal{X}_{V}^{2}: \vec{s}_{[n-1]} \geq \vec{t}_{[n-1]}\right\}\right)}_{=1 \text { by induction }}
\end{aligned}
$$




$$
\begin{aligned}
& \times(1-\underbrace{\nu\left(\left\{(\vec{s}, \vec{t}) \in \mathcal{X}_{V}^{2}: \vec{s}_{n}=0<\vec{t}_{n}=1 \mid \vec{s}_{[n-1]} \geq \vec{t}_{[n-1]}\right\}\right)}_{=0 \text { by definition of } \nu}) \\
& =1 .
\end{aligned}
$$

Hence

$$
\forall n \in \mathbb{N}: \quad \nu\left(\left\{(\vec{s}, \vec{t}) \in \mathcal{X}_{V}^{2}: \vec{s}_{[n]} \nsucceq \vec{t}_{[n]}\right\}\right)=0 .
$$

This implies that

$$
\nu\left(\left\{(\vec{s}, \vec{t}) \in \mathcal{X}_{V}^{2}: \vec{s} \nsupseteq \vec{t}\right\}\right)=0 .
$$

\subsection{A summary of the homogeneous case}

In the homogeneous case each of the sets defined in (3), after being identified with the respective cross-sections, reduces to a one-dimensional interval described by its non-trivial endpoint. The dominated Bernoulli parameter value (short: dominated value) of a $\mathrm{BPF} Y$ is

$$
\sigma(Y):=\max \left\{c: Y \stackrel{s t}{\geq} \Pi_{c}^{V}\right\} .
$$

For a non-empty class $C$ of BRFs this extends to

$$
\sigma(C):=\inf \{\sigma(Y): Y \in C\} .
$$

The critical domination values of a class $C$, assuming that $C(p)$ is non-empty for all $p$, are written as

$$
p_{\text {dom }}^{C}:=\inf \{p \in[0,1]: \forall Y \in C(p): \sigma(Y)>0\}
$$

and

$$
p_{\text {udom }}^{C}:=\inf \{p \in[0,1]: \sigma(C(p))>0\} .
$$

As the function $p \mapsto \sigma(C(p))$ is non-decreasing (29) the sets $\left.] p_{d o m}^{C}, 1\right]$ and ]$\left.p_{u d o m}^{C}, 1\right]$ are up-sets and we have the inequality

$$
p_{\text {dom }}^{C} \leq p_{\text {udom }}^{C} .
$$

The first known result is a bound on $p_{u d o m}^{\mathcal{C}_{G}^{\text {weak }}}$ in the homogeneous case, only depending on the maximal degree of $G$ :

Theorem 28 ([15, theorem 1.3]). If $G$ has uniformly bounded degree by a constant $D$, then

$$
p_{\text {udom }}^{\mathcal{C}_{G}^{\text {weak }}} \leq 1-\frac{(D-1)^{(D-1)}}{D^{D}}
$$

and for $p \geq 1-\frac{(D-1)^{(D-1)}}{D^{D}}$ the dominated parameter is uniformly minorated:

$$
\sigma\left(\mathcal{C}_{G}^{\text {weak }}(p)\right) \geq\left(1-\left(\frac{q}{(D-1)^{(D-1)}}\right)^{1 / D}\right)\left(1-(q(D-1))^{1 / D}\right) .
$$

Additionally

$$
\lim _{p \rightarrow 1} \sigma\left(\mathcal{C}_{G}^{\text {weak }}(p)\right)=1
$$


Recall that for $k \in \mathbb{N}_{0}$ the $k$-fuzz of $G=(V, E)$ is the graph with vertices $V$ and an edge for every pair of vertices at distance less than or equal to $k$ in $G$. Denote the $k$-fuzz of $\mathbb{Z}$ by $\mathbb{Z}_{(k)}$. Note that $\mathbb{Z}_{(k)}$ is $2 k$-regular. As $\mathbb{Z}_{(k)}$ has a natural order inherited from $\mathbb{Z}$ theorem 28 can be improved considerably:

Theorem 29 ([15, theorems 0.0, 1.5 and corollary 2.2$])$. On $\mathbb{Z}_{(k)}$ we have

$$
p_{\text {dom }}^{\mathcal{C}_{\mathbb{Z}_{(k)}}^{\text {weak }}}=p_{\text {udom }}^{\mathcal{C}_{\mathbb{Z}_{(k)}}^{\text {weak }}}=p_{\text {dom }}^{\mathcal{C}_{\mathbb{Z}_{(k)}}^{\text {strong }}}=p_{\text {udom }}^{\mathcal{C}_{\mathbb{Z}_{(k)}}^{\text {strong }}}=1-\frac{k^{k}}{(k+1)^{(k+1)}}
$$

For $p \geq p_{\text {udom }}^{\mathcal{C}_{\mathbb{Z}_{(k)}}^{\text {stron }}}$ the dominated parameter is minorated by

$$
\sigma\left(\mathcal{C}_{\mathbb{Z}_{(k)}^{\text {weak }}}(p)\right) \geq\left(1-\left(\frac{q}{k^{k}}\right)^{\frac{1}{k+1}}\right)\left(1-(q k)^{\frac{1}{k+1}}\right) .
$$

This implies a jump of $\sigma\left(\mathcal{C}_{\mathbb{Z}_{(k)}^{\text {weak }}}().\right)$ at the critical value $p_{\text {udom }}^{\mathcal{C}_{(k)}^{\text {weak }}}$, namely

$$
\forall k \in \mathbb{N}_{0}: \quad \frac{k}{(k+1)^{2}} \leq \sigma\left(\mathcal{C}_{\mathbb{Z}_{(k)}^{\text {weak }}}\left(p_{\text {udom }}^{\mathcal{C}_{\mathbb{Z}_{(k)}}^{\text {weak }}}\right)\right)
$$

To arrive at the equality in (53a) Liggett, Schonmann \& Stacey derived a lower bound from a particular probability measure, called Shearer's measure (see section 3). Furthermore it allowed them to show that

$$
\forall k \in \mathbb{N}_{0}: \quad \sigma\left(\mathcal{C}_{\mathbb{Z}_{(k)}}^{\text {strong }}\left(p_{\text {udom }}^{\mathcal{C}_{\mathbb{Z}_{(k)}}^{\text {strong }}}\right)\right) \leq \frac{k}{k+1}
$$

Thus our main result can be written as a corollary of theorems 2 and 3 :

Theorem 30. Let $G$ be a locally finite and connected graph. Then

$$
p_{\text {dom }}^{\mathcal{C}_{G}^{\text {weak }}}=p_{\text {udom }}^{\mathcal{C}_{G}^{\text {weak }}}=p_{\text {dom }}^{\mathcal{C}_{G}^{\text {strong }}}=p_{\text {udom }}^{\mathcal{C}_{G}^{\text {strong }}}=p_{\text {sh }}^{G} .
$$

If $G$ contains at least one infinite connected component and has uniformly bounded degree, then

$$
\sigma\left(\mathcal{C}_{G}^{\text {weak }}\left(p_{\text {udom }}^{\mathcal{C}_{G}^{\text {weak }}}\right)\right) \geq\left(q_{\text {udom }}^{\mathcal{C}_{G}^{\text {weak }}}\right)^{2}>0
$$

whereas if $G$ is finite we have

$$
\sigma\left(\mathcal{C}_{G}^{\text {weak }}\left(p_{\text {udom }}^{\mathcal{C}_{G}^{\text {weak }}}\right)\right)=0
$$

The discontinuity described in (55b) also holds for the more esoteric case of graphs having no uniform bound on their degree. In this case $p_{\text {udom }}^{\mathcal{C}_{G}^{\text {strong }}}=1$ and $\sigma\left(\mathcal{C}_{G}^{\text {weak }}(1)\right)=1>0$. An explanation for this discontinous transition might come from statistical mechanics, via the connection with hard-core lattice gases made by Scott \& Sokal [18]. It should be equivalent to the existence of a nonphysical singularity of the entropy for negative real fugacities for all infinite connected lattices. 


$$
\begin{aligned}
p_{s h}^{G} \stackrel{\text { (UD) }}{\geq} p_{\text {udom }}^{\mathcal{C}_{G}^{\text {weak }}} & \geq p_{\text {dom }}^{\mathcal{C}_{G}^{\text {weak }}} \\
\text { IV } & \text { IV } \\
p_{\text {udom }}^{\mathcal{C}_{G}^{\text {strong }}} & \geq p_{\text {dom }}^{\mathcal{C}_{G}^{\text {strong }}} \stackrel{(\text { ND) }}{\geq} p_{\text {sh }}^{G}
\end{aligned}
$$

Figure 2: Inequalities in the proof of (55). The four center inequalities follow straight from (51e) and (7). The inequality (ND) is an adaption of the approach used for $\mathbb{Z}_{(k)}$ in [15], while inequality (UD) is the novel interpretation of the optimal bounds of Shearer's measure.

The graph $\mathbb{Z}_{(k)}$ turns out to be a rare example of an infinite graph where we can construct Shearer's measure explicitely, in this case as a $(k+1)$-factor [16, section 4.2]. A second case immediately deducible from previous work would be the $D$-regular tree $\mathbb{T}_{D}$, where

$$
1-\frac{(D-1)^{(D-1)}}{D^{D}}=p_{s h}^{\mathbb{T}_{D}} \leq p_{d o m}^{\mathbb{T}_{D}} \leq 1-\frac{(D-1)^{(D-1)}}{D^{D}}
$$

by [19, theorem 2] and theorem 28 .

\subsection{Proofs of classical results}

The following proofs are given for completeness and to be able to underline the similarity with the stochastic domination proofs.

Proof. (of lemma 1) It is sufficient to prove (15b) inductively for one-vertex extensions. We prove (15) jointly by induction over the cardinality of $W$. The induction base for $W:=\{w\}$ is

$$
\mathbb{P}\left(Z_{w}=1\right)=p_{w}=\mu_{G, \vec{p}}\left(Y_{w}=1\right)=\Xi_{(\{w\}, \emptyset)}(\vec{p}) .
$$

In the induction step we extend $W$ to $\widetilde{W}:=W \uplus\{v\}$. Suppose that $\mu_{G, \vec{p}}\left(Y_{W}=\right.$ $\overrightarrow{1})=0$. Hence $\mu_{G, \vec{p}}\left(Y_{\widetilde{W}}=\overrightarrow{1}\right)=0$, too, and (15a) holds trivially. If $\mu_{G, \vec{p}}\left(Y_{W}=\right.$ $\overrightarrow{1})>0$, then $\mathbb{P}\left(Z_{W}=\overrightarrow{1}\right)>0$ by the induction hypothesis. Let $W \cap \mathcal{N}(v)=$ : $\left\{w_{1}, \ldots, w_{m}\right\}$ and $W_{i}:=W \backslash\left\{w_{i}, \ldots, w_{m}\right\}$. If $m=0$, then we revert to the equality in the induction base. If $m \geq 1$ then

$$
\begin{aligned}
& \mathbb{P}\left(Z_{v}=1 \mid Z_{W}=\overrightarrow{1}\right) \\
= & \frac{\mathbb{P}\left(Z_{v}=1, Z_{W}=\overrightarrow{1}\right)}{\mathbb{P}\left(Z_{W}=\overrightarrow{1}\right)} \\
\geq & \frac{\mathbb{P}\left(Z_{W}=\overrightarrow{1}\right)-q_{v} \mathbb{P}\left(Z_{W \backslash \mathcal{N}(v)}=\overrightarrow{1}\right)}{\mathbb{P}\left(Z_{W}=\overrightarrow{1}\right)} \\
= & 1-\frac{q_{v}}{\prod_{i=1}^{m} \mathbb{P}\left(Z_{w_{i}}=\overrightarrow{1} \mid Z_{W_{i}}=\overrightarrow{1}\right)} \\
\geq & 1-\frac{q_{v}}{\prod_{i=1}^{m} \alpha_{W_{i}}^{w_{i}}(\vec{p})} \\
= & \alpha_{W}^{v}(\vec{p})
\end{aligned}
$$$$
\text { as } Z \in \mathcal{C}_{G}^{\text {weak }}(\vec{p})
$$$$
\text { induction hypothesis as }\left|W_{i}\right|<|W|
$$$$
\text { using the fundamental identity (31) }
$$ 
This proves (15b). For (15a) see that

$$
\begin{aligned}
\mathbb{P}\left(Z_{\widetilde{W}}=\overrightarrow{1}\right)=\mathbb{P}\left(Z_{v}=1 \mid Z_{W}=\overrightarrow{1}\right) \mathbb{P} & \left(Z_{W}=\overrightarrow{1}\right) \\
& \geq \alpha_{W}^{v}(\vec{p}) \mu_{G, \vec{p}}\left(Y_{W}=\overrightarrow{1}\right)=\mu_{G, \vec{p}}\left(Y_{\widetilde{W}}=\overrightarrow{1}\right) .
\end{aligned}
$$

Proof. (of theorem 5) Assume that $q \leq \frac{(D-1)^{(D-1)}}{D^{D}}$. We claim that for every escaping $(W, v)$ (see definition 13$)$

$$
\alpha_{W}^{v}(p) \geq 1-\frac{1}{D}
$$

This claim implies that $\Xi_{G(W)}(p) \geq\left(\frac{D-1}{D}\right)^{|W|}>0$ for every finite $W \subseteq V$. Hence $p \geq p_{s h}^{G}$. We prove the claim (56) by induction over the cardinality of $W$. The induction base is given by

$$
\alpha_{\emptyset}^{v}(p)=p \geq 1-\frac{(D-1)^{(D-1)}}{D^{D}} \geq 1-\frac{1}{D} .
$$

As $(W, v)$ is escaping $v$ has at most $m \leq D-1$ neighbours in $W$, which we denote by $\left\{w_{1}, \ldots, w_{m}\right\}:=W \cap \mathcal{N}(v)$. Using the fundamental identity (31) and (56) the induction step is

$$
\begin{aligned}
& \alpha_{W}^{v}(p)=1-\frac{q}{\prod_{i=1}^{m} \alpha_{W \backslash\left\{w_{i}, \ldots, w_{m}\right\}}^{w_{i}}(p)} \\
& \quad \geq 1-\frac{q}{\prod_{i=1}^{m}\left(1-\frac{1}{D}\right)} \geq 1-\frac{q}{\left(\frac{D-1}{D}\right)^{D-1}} \geq 1-\frac{1}{D} .
\end{aligned}
$$

